\title{
Trickle-based Interference Cancellation Schemes for CDMA Systems
}

\author{
Pooyan Haghighat
}

\author{
A Thesis \\ in \\ The Department \\ of \\ Electrical and Computer Engineering
}

Presented in Partial Fulfillment of the Requirements

for the Degree of Master of Applied Science

Concordia University

Montreal, Quebec, Canada

August 2008

(C) Pooyan Haghighat, 2008 


$\begin{array}{ll}\begin{array}{l}\text { Library and } \\ \text { Archives Canada }\end{array} & \begin{array}{l}\text { Bibliothèque et } \\ \text { Archives Canada }\end{array} \\ \begin{array}{l}\text { Published Heritage } \\ \text { Branch }\end{array} & \begin{array}{l}\text { Direction du } \\ \text { Patrimoine de l'édition }\end{array} \\ \begin{array}{l}\text { 395 Wellington Street } \\ \text { Ottawa ON K1A 0N4 } \\ \text { Canada }\end{array} & \begin{array}{l}\text { 395, rue Wellington } \\ \text { Ottawa ON K1A 0N4 } \\ \text { Canada }\end{array}\end{array}$

Your file Votre référence ISBN: 978-0-494-45704-7 Our file Notre référence ISBN: 978-0-494-45704-7

NOTICE:

The author has granted a nonexclusive license allowing Library and Archives Canada to reproduce, publish, archive, preserve, conserve, communicate to the public by telecommunication or on the Internet, loan, distribute and sell theses worldwide, for commercial or noncommercial purposes, in microform, paper, electronic and/or any other formats.

The author retains copyright ownership and moral rights in this thesis. Neither the thesis nor substantial extracts from it may be printed or otherwise reproduced without the author's permission.
AVIS:

L'auteur a accordé une licence non exclusive permettant à la Bibliothèque et Archives Canada de reproduire, publier, archiver, sauvegarder, conserver, transmettre au public par télécommunication ou par l'Internet, prêter, distribuer et vendre des thèses partout dans le monde, à des fins commerciales ou autres, sur support microforme, papier, électronique et/ou autres formats.

L'auteur conserve la propriété du droit d'auteur et des droits moraux qui protège cette thèse. $\mathrm{Ni}$ la thèse ni des extraits substantiels de celle-ci ne doivent être imprimés ou autrement reproduits sans son autorisation.
In compliance with the Canadian Privacy Act some supporting forms may have been removed from this thesis.

While these forms may be included in the document page count, their removal does not represent any loss of content from the thesis.
Conformément à la loi canadienne sur la protection de la vie privée, quelques formulaires secondaires ont été enlevés de cette thèse.

Bien que ces formulaires aient inclus dans la pagination, il n'y aura aucun contenu manquant.

\section{Canada}




\section{Abstract \\ Trickle-based Interference Cancellation Schemes for CDMA Systems}

Pooyan Haghighat

In this thesis, we introduce a novel approach to interference cancellation for code division multiple access uplink transmission. Several models combining principles of serial and parallel interference cancellation are discussed. The proposed scheme is derived from the analysis of these hybrid models and applies a user configuration algorithm (termed "trickle") in order to provide an improved bit-error-rate performance. The algorithm utilizes an adaptive matrix to compute the required configuration to be used for the subsequent interference cancellation stage. Bit-streaming, pipelined multiuser detection is employed and channel estimates are obtained using sample pilot data known at the receiver. We demonstrate that significant performance improvements can by achieved over various hybrid schemes. A reduced-complexity version of the trickle algorithm is also introduced where the processing delay is greatly reduced while maintaining similar performance. We present several numerical examples through which we demonstrate the efficacy of the proposed algorithms relative to existing interference cancellation algorithms. 
To my mother and father, and all those who have helped me along the way... 


\section{Contents}

List of Figures $\quad$ vii

List of Tables $\quad$ ix

List of Symbols $\quad$ ix

List of Acronyms $\quad$ xi

1 Introduction $\quad 1$

1.1 Motivation for Study and Problem Statement . . . . . . . . . . 2

1.2 Overview of Multiple Access Methods . . . . . . . . . . . . . . . . 3

1.3 Thesis Contribution . . . . . . . . . . . . . . . . . 4

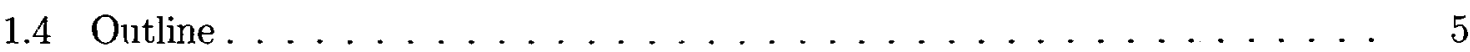

2 Literature Review $\quad 7$

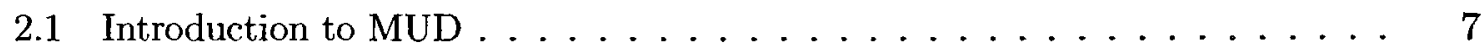

2.2 Single User Model . . . . . . . . . . . . . . . . . . . 7

2.2.1 Spreading Sequences

2.2 .2 Typical DS-SS Receivers . . . . . . . . . . . . . . 9

2.2 .3 Correlation and RAKE Receivers . . . . . . . . . . 10

2.3 Multiuser Detectors . . . . . . . . . . . . . . . . . . . . 11

2.3 .1 Optimal Detection $\ldots \ldots \ldots \ldots \ldots \ldots$

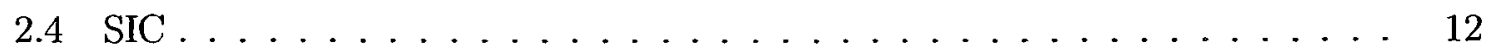

2.4 .1 Conventional SIC . . . . . . . . . . . . . . . 12

2.4 .2 Soft-decision $\mathrm{SIC} \ldots \ldots \ldots \ldots \ldots$ 
2.4 .3 Power Control for SIC . . . . . . . . . . . . . . . . 14

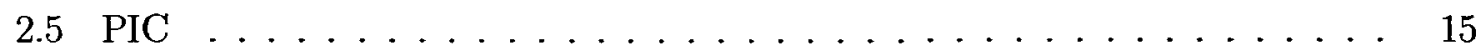

2.5 .1 Conventional PIC . . . . . . . . . . . . . 15

2.5 .2 Partial PIC . . . . . . . . . . . . . . 16

2.5.3 Frequency Diversity Combining with PPIC . . . . . . . 17

2.5 .4 Iterative PIC $\ldots \ldots \ldots \ldots \ldots \ldots \ldots$

2.6 Hybrid IC . . . . . . . . . . . . . . . . . . . . 18

2.6 .1 Concatenated IC $\ldots \ldots \ldots \ldots \ldots$

2.6 .2 Groupwise IC . . . . . . . . . . . . . . . . . 20

2.7 Introduction to Pipelined Multiuser Detection . . . . . . . . . . . 21

2.7.1 Background to the Partial Parallel Pipelined Multiuser Detector . . . 21

2.7.2 Channel Estimation and Pipelined Multistage Detector . . . . . . 21

2.7.3 Matched Filter and Pipelined Multistage Detector Overview .... . 22

2.7.4 Detailed System Model Description . . . . . . . . . . . . 22

3 Proposed Scheme - The Trickle Algorithm 27

3.1 Derivation of the Trickle Algorithm . . . . . . . . . . . . 27

3.2 Exponentially Varying Group Sizes . . . . . . . . . . . . . . . 28

3.3 Description of the Trickle Algorithm . . . . . . . . . . . . 30

3.4 Improved Trickle Algorithm . . . . . . . . . . . . . . 33

4 Results $\quad 35$

4.1 Exponential Versus Inverse Exponential . . . . . . . . . . . . . 35

4.2 Performance Comparison between Trickle, Linear, Exponential, MF, PIC, and

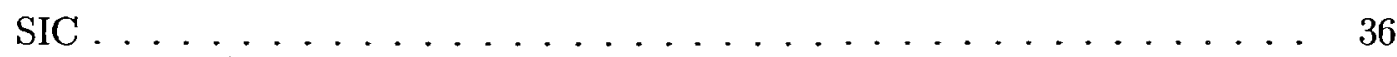

4.3 Processing Delay Reduction Analysis . . . . . . . . . . . . . . 38

5 Conclusion and Future Work $\quad 42$

5.1 Conclusion . . . . . . . . . . . . . . . . . 42

5.2 Future work . . . . . . . . . . . . . . . . . 42 


\section{List of Figures}

1.1 Overview of MUD models . . . . . . . . . . . . . . . . . 2

1.2 Summary of multiple access methods . . . . . . . . . . . . . 4

2.1 Single-riser DS-SS model . . . . . . . . . . . . . . . . . 7

2.2 A BPSK-based conventional matched filter DS-SS receiver $\ldots \ldots \ldots$

2.3 BPSK-based RAKE CDMA Receiver . . . . . . . . . . . . . . . 11

2.4 Uplink transmission at base station receiver $\ldots \ldots \ldots \ldots \ldots$

2.5 Successive Interference Cancellation . . . . . . . . . . . . . 13

2.6 BER plot of SIC receiver for $K=5 \ldots \ldots \ldots \ldots \ldots \ldots \ldots$

2.7 BER plot of SIC receiver for $K=25 \ldots \ldots \ldots \ldots \ldots \ldots$

2.8 Parallel Interference Cancellation $\ldots \ldots \ldots \ldots \ldots$

2.9 BER plot for single-stage PIC for $K=20 \ldots \ldots \ldots \ldots \ldots \ldots$

2.10 BER plot for 3 -stage PIC for $K=20 \ldots \ldots \ldots \ldots \ldots \ldots \ldots$

2.11 Flowchart of serial-parallel receiver model f . . . . . . . . . . . 19

2.12 Block diagram of group-wise interference cancellation scheme for $K=5$. . . 20

2.13 Pipelined detection process . . . . . . . . . . . . . . . . 24

2.14 Multistage multiuser detection overview $\ldots \ldots \ldots \ldots \ldots$

3.1 Block diagram of inverse exponential group-wise scheme for $K=8$. . . . 29

3.2 Block diagram of exponential group-wise scheme for $K=8 \ldots \ldots \ldots$

3.3 Sample $\mathrm{Q}$ matrix computation for 7 users. . . . . . . . . . . . . . 32

3.4 Sample Q matrix for $K=21 . \ldots \ldots \ldots \ldots \ldots \ldots \ldots$

3.5 Reduced Q matrix for $K=21 \ldots \ldots \ldots \ldots \ldots \ldots \ldots \ldots \ldots$

4.1 BER comparison between exponential and inverse exponential group-wise schemes for $K=20 . \ldots \ldots \ldots \ldots \ldots \ldots \ldots \ldots \ldots$ 
4.2 BER comparison between linear, exponential, MF, SIC, 3-stage PIC, and trickle algorithm for $K=20 \ldots \ldots \ldots \ldots \ldots \ldots$. . . . . . . . 37

4.3 BER comparison between linear, exponential, MF, SIC, 3-stage PIC, and

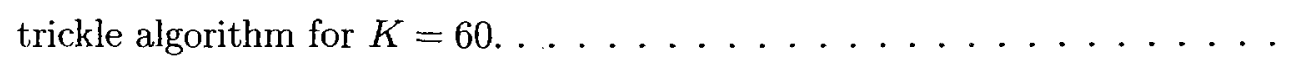

4.4 BER comparison between linear, exponential, MF, SIC, 3-stage PIC, and trickle algorithm for $K=120 \ldots \ldots \ldots \ldots \ldots \ldots$

4.5 BER comparison between improved and standard trickle algorithmfor $K=20.40$

4.6 Percent reduction in number of IC stages and processing events for the improved trickle algorithm. . . . . . . . . . . . . . . . 41 


\section{List of Symbols}

$\begin{array}{ll}b(t) & \text { input data sequence for DS-SS transmitter } \\ b_{i} & \text { data bit for } i \text { th user } \\ r_{i} & \text { vector form of received signal for } i \text { th user for multiuser detector } \\ \hat{b}_{i} & \text { estimated data bit for } i \text { th user } \\ c(t) & \text { pseudo-noise code waveform } \\ c_{j} & \text { code chip for } j \text { th user } \\ \mathrm{Q} & \text { user configuration matrix utilized by the trickle algorithm } \\ \hat{\mathbf{Q}} & \text { reconfiguration of Q matrix with first two rows inverted } \\ K & \text { number of users } \\ l & \text { multipath component index } \\ L_{P} & \text { number of the multipath components of the fading channel } \\ M & \text { number of bits per frame } \\ U & \text { number of users to be added to a given PIC stage } \\ s & \text { index of a given PIC stage } \\ N & \text { spreading factor } \\ P & \text { transmit power } \\ r_{p}(t) & \text { additive white Gaussian noise } \\ r(t) & \text { composite signal at input of the } p \text { th PIC stage } \\ T & \text { symboriming signal at the receiver } \\ & \end{array}$


$T_{c}$

$\operatorname{diag}\{\cdot\}$

$Z_{i}$ number of initial rows in $\mathbf{Q}$ matrix losing a single iteration

number of initial rows in $\mathbf{Q}$ matrix amongst which iterations are redistributed

remainder of iterations redistributed to first row in $\mathrm{Q}$ matrix

number of redistributions carried out by the trickle algorithm matched filter output

cross correlation matrix of the synchronized spreading codes

diagonal matrix containing the amplitudes of the users

lower triangular portion of matrix $R$

number of received bits

data bit for $i$ th user for pipelined multiuser detector

estimated data bit for $i$ th user

partial correlation matrix for past and future bits of interfering users correlation matrix of current bits of interfering bits

signal-to-noise ratio

phase shift

carrier frequency

finite propagation delay

estimated $\phi$

estimated $\theta$

estimated $\tau$

matrix hermitian transpose operation

matrix tanspose operation

code chip interval

diagonalization operation

decision metric for the $i$ th user 


\section{List of Acronyms}

3G

AMPS

AWGN

BPSK

BER

CDMA

DS-SS

EETACS

FDMA

FDC

GSIC

GSM

IGPIC

IGSIC

MAI

MF

ML

MUI

NTT

PDC

PIC

PPIC

PN third generation

advanced mobile phone service

additive white Gaussian noise

binary phase shift keying

bit error rate

code division multiple access

direct-sequence spread spectrum

extended european total access cellular system

frequency division multiplexing access

frequency diversity combining

group-wise successive interference cancellation

group special mobile

iterative group-wise parallel interference cancellation

iterative group-wise serial interference cancellation

multiple access interference

matched filter

maximum likelihood

multiuser detection

Nippon telephone and telegraph

pacific digital cellular

parallel interference cancellation

partial parallel interference cancellation

pseudo noise 
SNR

signal-to-noise ratio

SIC

successive interference cancellation

TDMA

time division multiplexing access

USDC

United States digital cellular

W-CDMA

wideband code division multiple access 


\section{Chapter 1}

\section{Introduction}

Today's world of cellular communication is faced with the difficult challenge of supporting the needs of wireless applications such as video, text messaging, and web browsing, etc.. This has led to an increasing demand for reliable high-rate data communication. The work of Nilsson [1] illustrates how first generation analog systems as well as second generation digital systems are geared towards providing reliable communication specific to voice application traffic. Third generation (3G) systems, more specifically systems based on Code Division Multiple Access (CDMA) technology, are geared towards significantly higher rate applications and face greater challenges concerning capacity as compared to their predecessors. The most significant of these challenges involves multiple-access interference (MAI). Considered one of the major limiting factors in wireless communication, MAI primarily stems from the asynchronous nature of the uplink channel where orthogonality between the spreading sequences of different users cannot be guaranteed. Reducing MAI can significantly improve the overall spectral efficiency of the system. This can be achieved by means of multiuser detection (MUD) where interference caused by time offsets and fading are jointly estimated for all users to provide better detection for individual users. Much of the recent research in the field of telecommunications has focused on MUD and IC techniques designed to improve performance through MAI reduction [2]-[39].

MUD techniques can be divided into two categories: Linear MUD and subtractive interference cancellation detectors [19]. The former involves an iterative process where linear mapping is utilized to produce a new set of outputs, and the latter iteratively subtracts interference from the received signal through estimation. Previous work [2]-[4] has shown that optimum detection schemes are impractical due to their extremely high complexity. Thus, sub-optimal IC receiver structures have been the focus of much of the ensuing research concerning MUD [5]-[27]. These receivers can be further divided into two subcategories: 
successive interference cancellation (SIC) and parallel interference cancellation (PIC). Past studies [32]-[11] describe the merits of hybrid interference cancellation techniques that combine the advantages of both SIC and PIC, several models of which are proposed in [33], [11]. An overview of MUD and IC techniques is shown below in Fig. 1.1.

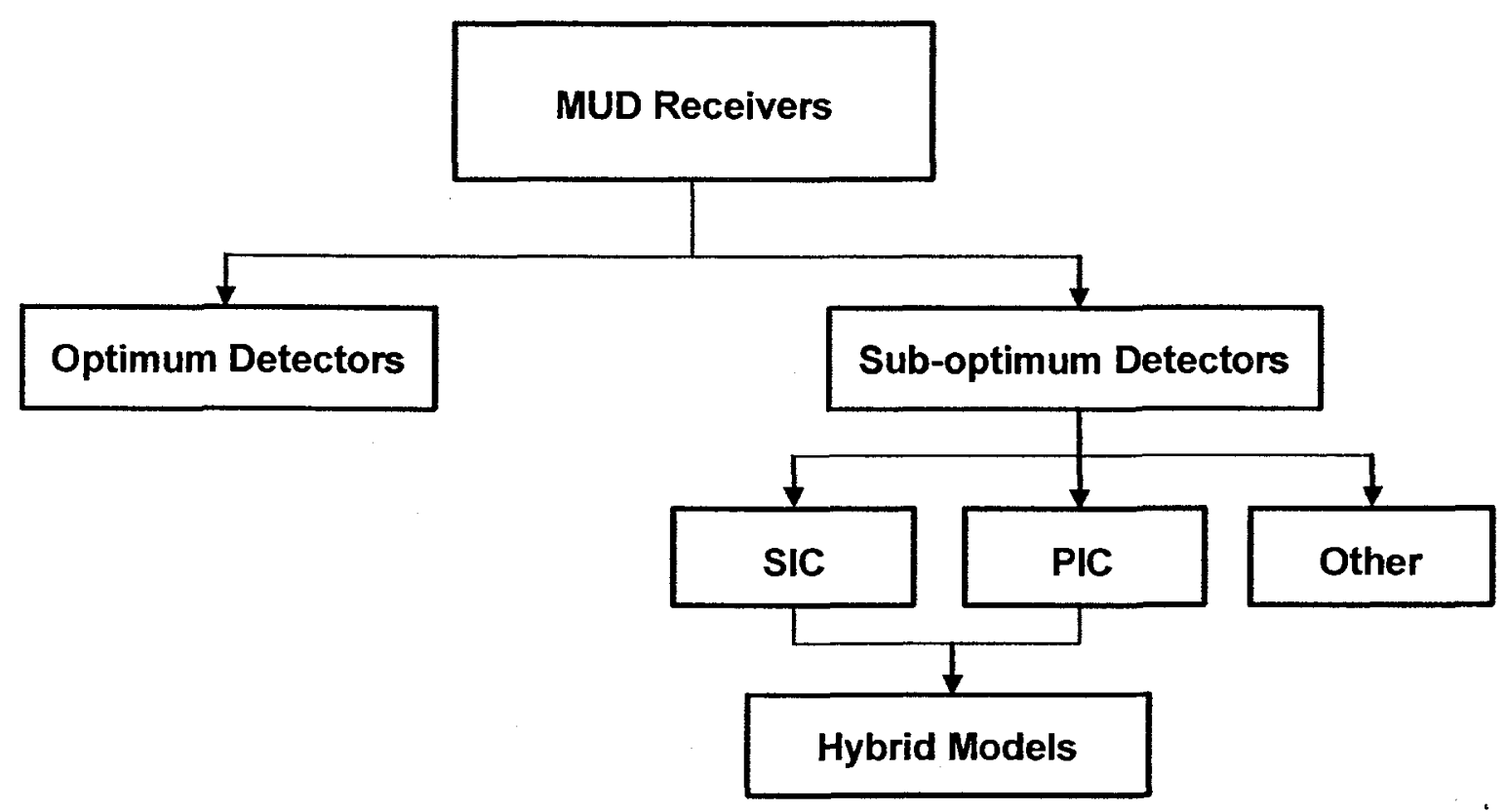

Figure 1.1: Overview of MUD models

The proposed "trickle" algorithm presented in this thesis is a hybrid IC scheme implementing a reconfiguration of users in order to achieve more reliable bit error rate (BER) performance as compared to standard SIC and PIC schemes.

\subsection{Motivation for Study and Problem Statement}

The optimal multiuser receiver algorithm for CDMA derived by Verdu [2] is the foundation of much of the research conducted on sub-optimal receiver algorithms and iterative detection techniques [3].

The aim of this study is to develop a sub-optimum receiver capable of providing improved BER performance while minimizing cost in processing delay and complexity. More specifically, the focus is on developing a hybrid receiver capable of combing benefits inherent to both SIC and PIC receviers. Previous work in this area has provided user configuration 
schemes designed arbitrarily [33],[11], albeit achieving improved BER performance as compared to conventional schemes [4]. This study looks to optimize these models and provide an adaptive algorithm capable of adjusting to any given number of users. The following guidelines were respected in order to achieve these aims:

- The Theoretical Stage - Research into past MUD structures and current hybrid IC schemes as well as related theory was conducted.

- The Practical Stage - The performance of the hybrid schemes was investigated with regards to factors such as complexity, delay, and BER.

- The Formulation Stage - New algorithms stemming from previous work were designed; the emphasis was put on BER performance.

- The Conclusion Stage - Evaluation of the proposed hybrid IC scheme is made through extensive computer simulations.

\subsection{Overview of Multiple Access Methods}

In Frequency Division Multiple Access (FDMA), distinct frequency channels are assigned to each transmitter while receivers select the appropriate incoming signal by tuning to the desired frequency [43]. It was most predominantly seen in the first generation of cellular systems such as the Advanced Mobile Phone Service (AMPS), the Extended European Total Access Cellular System (ETACS), and the Nippon Telephone and Telegraph (NTT) system [45]. Second generation cellular systems generally employed Time Division Multiple Access (TDMA) as a multiple access technique. In contrast to FDMA, TDMA separates users by assigning each user a short time slot to transmit. Thus, users communicate one by one in a cyclic fashion. Second generation systems also include the Group Special Mobile (GSM) standard, the United States Digital Cellular (USDC) standard (also known as the Intermediate Standard-54, or IS-54), and the Pacific Digital Cellular (PDC) standard. However, for comparison purposes, these latter standards are not included in the discussion.

Finally, CDMA emerged as one of the leading standards in the latter stages of development of second generation cellular systems. Also known as Intermediate Standard-95 (IS-95), CDMA is based on spread spectrum technology, where each user is assigned a unique spreading code which is used by the receiver in order to differentiate between the desired incoming signal and incoming signals from all other users. Contrary to FDMA and TDMA where users are orthogonalized along frequency and time, CDMA signals overlap in both time and frequency, but are distinguishable by their signature codes (i.e. the codes in CDMA). For each user, the code signal multiplies the data signal, and since the code rate is much higher 
than the data rate, the resulting signal bears a higher rate and hence a "spreaded spectrum" compared to the data rate [40].

An overview of the abovementioned multiple access methods is shown below in Fig. 1.2

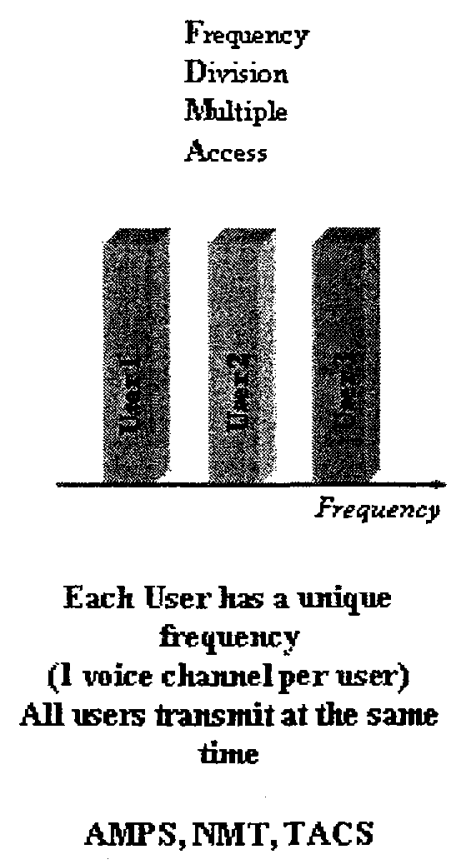

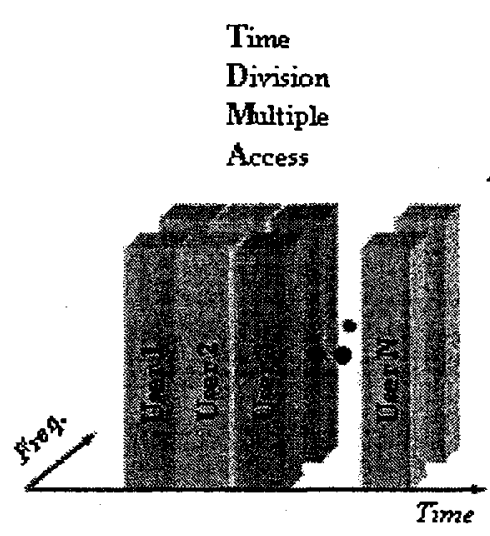

Each User has a wique time slot

Each Data Chamuel has a unique position within the time slot Several users share the same frequency

IS-136, GSM, PDC

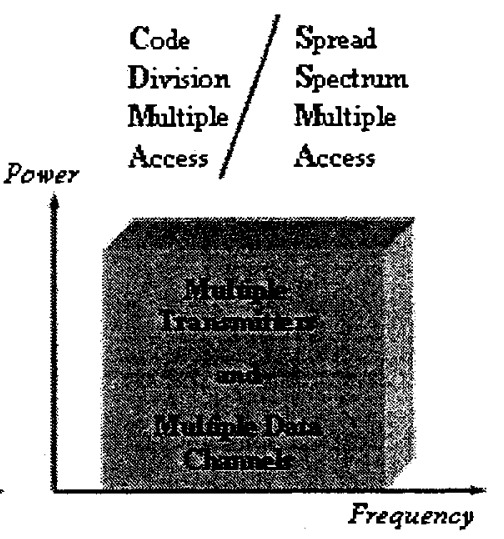

Each Transmither has a unique spreading code Each Data Charuel has a unique Orthogonal Code Many users share the same frequency and time

IS-95, CDMA 2000, WCDMA

Figure 1.2: Summary of multiple access methods

The user blocks in the diagram illustrate the efficiency of the use of the medium available by the various multiple access methods. This study presents a novel interference cancellation scheme for CDMA uplink communication. Derivation and description of the model is discussed in the following sections.

\subsection{Thesis Contribution}

The main contributions of this thesis are:

- Study and evaluation of various multiuser detection algorithms for interference cancellation receivers, including conventional SIC and PIC schemes. The results demonstrate how performance degrades as the number of users is increased due to a corresponding increase in MAI power. Ideal power distribution is assumed. 
- Introduction to exponential and inverse exponential hybrid models derived from hybrid model consisting of linearly varying group sizes. It is observed through simulation that the exponential group-wise structure offers improved channel estimation for higher values of SNR and consequently demonstrates better BER performance than the inverse exponential model. Estimation is further improved through additional iterations for users with strong signal strength, leading to the proposed scheme.

- Development of a new multiuser detection algorithm for interference cancellation termed "trickle". This algorithm is based on the exponential group-wise model, utilizing an adaptive matrix to compute the required configuration of users for the interference cancellation stages involved. Detailed description of the various parameters of the trickle scheme are also given.

- Study of the bit-streaming, pipelined MUD receiver structure. The model incorporates a pipelined MUD scheme to obtain channel estimates and carry out the IC process. The study provides description of the system model chosen for simulation the proposed scheme. Originally proposed in [21], alternative models could be employed to carry out the channel estimation process.

- Evaluation of the performance of the trickle algorithm. Through simulation, comparisons are made to SIC, PIC, as well as standard linear and exponential hybrid receiver structures. The performance results demonstrate a significant improvement over the aforementioned schemes.

- Development of an improved trickle algorithm. A reduction in the total number of IC stages is carried out while maintaining performance. This is achieved by limiting each distinct grouping of users to a maximum of three stages of IC. Timing analysis for the reduced trickle scheme is provided and it is shown that a significant decrease in processing delay is achieved at the cost of a minimal degradation of the BER performance.

\subsection{Outline}

A comprehensive look at MUD techniques is given in Chapter 2, beginning from the pimitive single-user receiver structures up to the more recent MUD receivers. Emphasis is put on interference cancellation receivers, while several hybrid IC models are discussed in greater detail. Simulations for conventional IC models are also provided. Comparisons between 
various structures are made throughout the chapter underlining advantages and disadvantages inherent to the schemes discussed, providing insight to the motivation for the proposed "trickle" algorithm.

A detailed description of the pipelined MUD receiver structure employed in this thesis is given as well in order to allow for the proper duplication of the results obtained. The scheme in question is a means of simulation for PIC receivers and its inclusion in this study is arbitrary. Its parameters are nonetheless presented as its benefits are demonstrated in corresponding simulation results. All relevant equations pertaining to the scheme are included.

Chapter 3 includes the derivation of the proposed algorithm, descriptions of both the exponential and inverse exponential models, as well as a detailed breakdown of the "trickle" algorithm along with the system parameters involved in the design of the improved "trickle" scheme. A brief analysis of the gain in processing delay is also given in this chapter.

In Chapter 4, simulation results of the proposed algorithm are compared to that of various conventional IC and hybrid schemes. As well, a more detailed analysis of the reduction in processing delay is given.

Finally, the conclusion to the thesis is presented in Chapter 5 with future work suggestions. 


\section{Chapter 2}

\section{Literature Review}

\subsection{Introduction to MUD}

As was previously mentioned, a wide range of MUD techniques have been investigated in the past. In this section, we investigate the interference cancellation subclass of MUD receivers, presenting the original multiple-access (uplink) schemes designed to achieve superior performance as compared to single-user detectors, as well as subsequent schemes designed to overcome the shortcomings and limitations of their ancestors.

\subsection{Single User Model}

Let us consider the direct-sequence spread spectrum (DS-SS) transmitter model for a single user with BPSK digital modulation shown below in Figure 2.1,

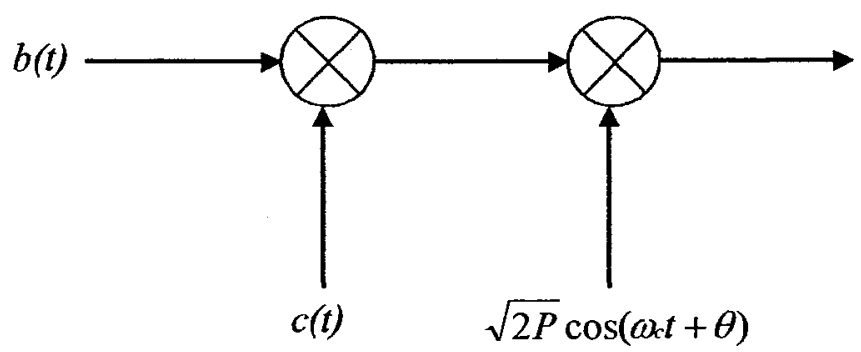

Figure 2.1: Single-user DS-SS model

where $b(t)$ is given by

$$
b(t)=\sum_{i=-\infty}^{\infty} b_{i} \Lambda_{T}(t-i T)
$$


$\left\{b_{i}\right\}$ is a set of independent identically distributed Bernouili random variables and $b_{i}$ is the $i$ th data bit taking on values of \pm 1 with equal probability. $\Lambda_{T}(t)$ is the applied rectangular pulse shape given by

$$
\Lambda_{T}(t)=\left\{\begin{array}{c}
1,0 \leq t \leq T \\
0, \text { otherwise }
\end{array}\right.
$$

where $T$ is the duration of the data bit.

Similarly, the pseudo-noise (PN) code waveform $c(t)$ is given by

$$
c(t)=\sum_{j=1}^{M} c_{j} \Lambda_{T c}\left(t-j T_{c}\right),
$$

where $M$ is the number of bits per frame, $c_{j}$ and $b_{i}$ are identically distributed, $\left\{c_{j}\right\}$ is set of randomly generated spreading codes of length $N$, where $N$ is the spreading factor, while the elements of $\left\{c_{j}\right\}$ are referred to as code chips, and $T_{c}$ is the code chip interval where $T=N T_{\mathrm{c}}$.

The resulting basic transmitted spread signal $s(t)$ is expressed as

$$
s(t)=\sqrt{2 P} b(t) c(t) \cos \left(\omega_{c} t+\theta\right)
$$

where $\omega_{c}$ and $\theta$ are the carrier frequency and phase, respectively.

\subsubsection{Spreading Sequences}

System performance is directly affected by the configuration of PN codes. Under ideal conditions, PN codes allow multiple users to share bandwidth without interfering with one another. However, it is impractical to achieve such performance and codes with near-ideal properties are used. The properties in question refer to the auto-correlation and crosscorrelation of the sequences. PN codes are designed to minimize auto-correlation for nonzero delay and also to minimize cross-correlation over all delays [2]. These factors directly affect the amount of interference between users of the system. In other words, how greatly the PN codes approach the optimal levels for both auto- and cross-correlation determines how greatly users interfere with one another, and in turn in large part determines system performance.

In general, random codes demonstrate good auto- and cross-correlation properties, which is why they are employed by the majority of DS-SS systems. Some of the more common types of PN codes are Maximal Length Sequences or m-sequences. Other types of PN codes can be derived from m-sequences such as Gold codes and Kasami Sequences, Scholtz, [19]. Each 
type of code has a particular advantage and depending upon system requirements, various performance parameters can be traded-off by the use of one type of code over another [4]. The system model for the propsed algorithm employs Gold codes and is described in greater detail in subsequent sections.

\subsubsection{Typical DS-SS Receivers}

\section{Matched Filter Receiver}

The matched filter (MF) receiver is the basis of a significant set of various receiver structures available. Once the signal is transmitted through an AWGN channel, the receiver generates bit decisions which are affected by channel conditions and the detection algorithm employed in the previously mentioned PN code properties. The incoming transmitted signal at the receiver is given by

$$
r(t)=s(t-\tau)+n(t)
$$

where $s(t)$ represents the original transmitted signal, $\tau$ is the finite propagation delay aquired during transmission, and $n(t)$ is a randomly generated additive white Gaussian noise (AWGN).

By expanding (2.4) and incorporating (2.3) we obtain

$$
r(t)=\sqrt{2 P} b(t-\tau) c(t-\tau) \cos \left(\omega_{c}(t-\tau)+\theta\right)
$$

The detection process consists of reversing the sequence of operations executed at the transmitter: demodulation, dispreading, and bit decisions. Demodulation involves synchronization between the receiver's and transmitter's respective carrier frequencies. Once the receiver has achieved carrier lock, the receiver then regenerates a synchronous copy of the PN code waveform (2.2) to despread the incoming signal. The signal then passes through a correlator which produces a decision metric given by

$$
Z_{i}=\int_{i T+\tau}^{(i+1) T+\tau} r(t) c(t-\tau) \cos \left(\omega_{c}(t-\tau)+\theta\right) d t
$$

The final step involves a non-linear threshold device modelled as a hard limiter, which yields an estimate of the transmitted bit $\hat{b}_{i}$ where 


$$
\hat{b}_{i}=\left\{\begin{array}{r}
1, Z_{i} \geq 0 \\
-1, Z_{i}<0
\end{array}\right.
$$

The basic diagram of a BPSK-based conventional matched filter DS-SS receiver is shown below in Fig. 2.2 below.

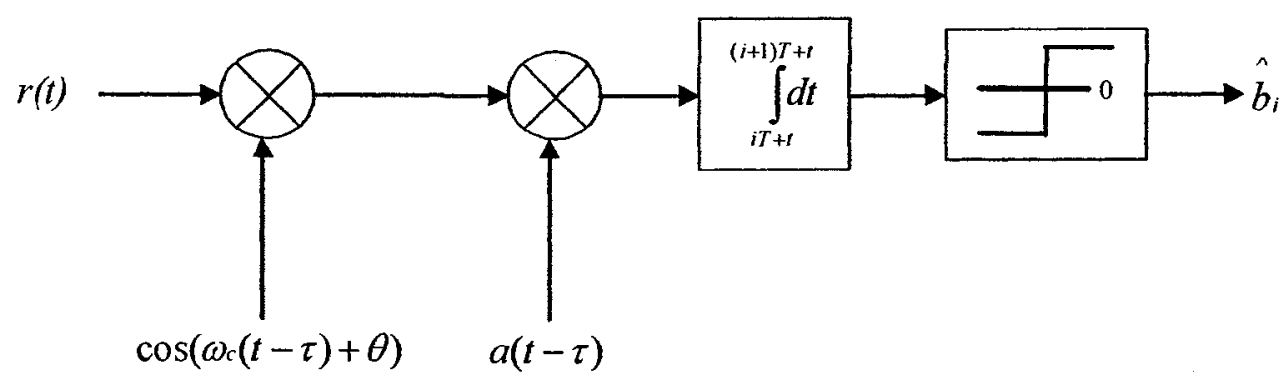

Figure 2.2: A BPSK-based conventional matched filter DS-SS receiver

Previous studies [2], [9] have shown that the matched filter receiver achieves the highest BER performance when attempting to detect a known signal.

\subsubsection{Correlation and RAKE Receivers}

The conventional CDMA receiver structure consists of a bank of DS-SS receivers each of which are associated with a single user. Bit estimates are formed from the outputs of the correlation receiver. Proposed for use in the IS-95 standard, the relatively low complexity of the receiver is countered to a certain extent by relatively poor performance. The RAKE receiver, introduced by [9], takes advantage of the inherent multipath diversity in a frequency selective fading CDMA channel. Due to the much smaller coherence bandwidth of the channel as compared to the bandwidth of the transmitted wideband CDMA signal, arriving multipath signals are seperately and effectively processed [43]. This is achieved by utilizing several sub-receivers each tuned to individual multipath components. The RAKE receiver structure is showin in Fig. 2.3 below.

The receiver is named RAKE due to its similarity to a garden rake, where each finger is capable of tracking the multipath components of each user. The RAKE receiver scheme is similar to that of the correlation receiver with the added RAKE combiner process. 


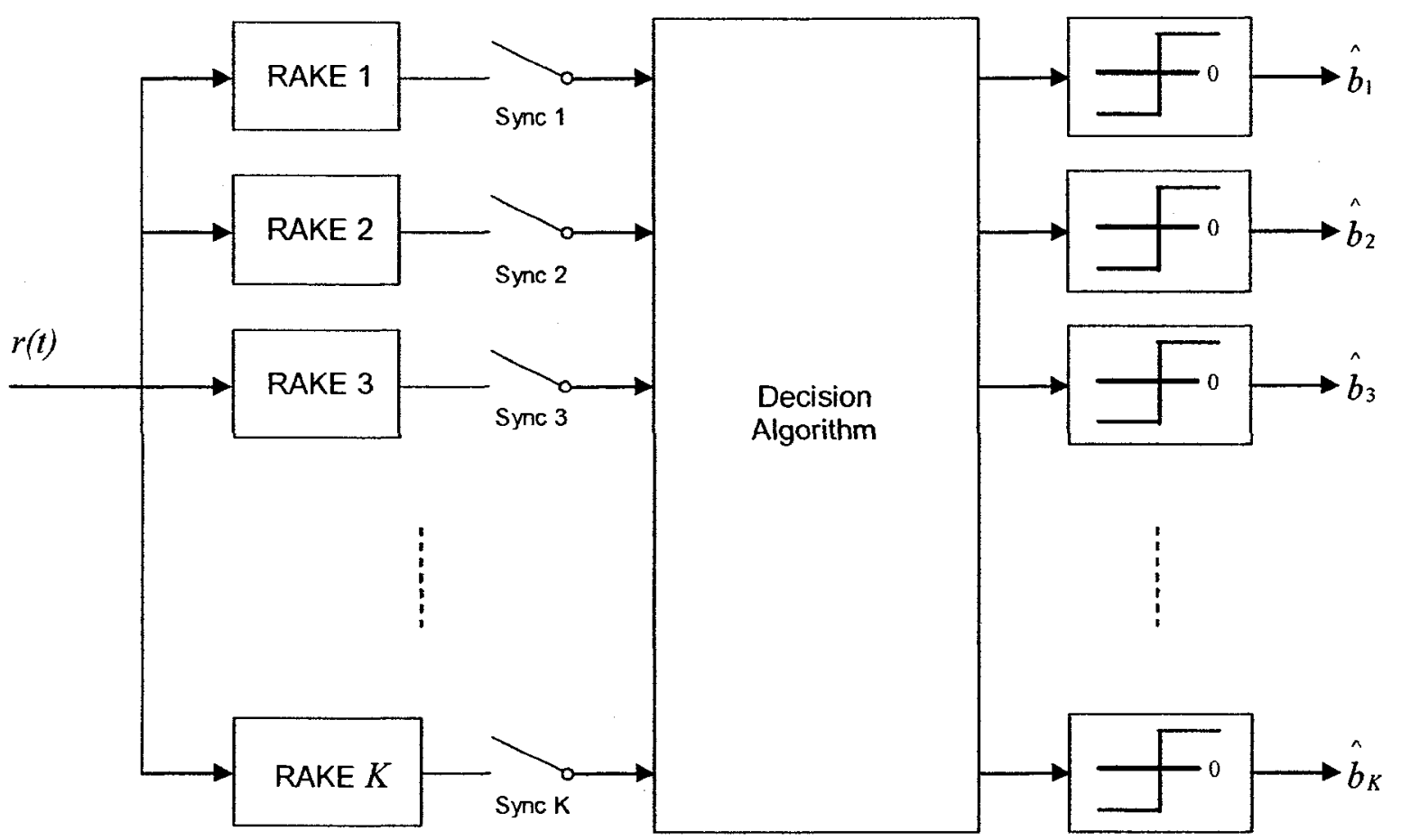

Figure 2.3: BPSK-based RAKE CDMA Receiver

\subsection{Multiuser Detectors}

Single user detectors are not optimal for CDMA or WCDMA due to the manner in which they treat interference from other users as channel noise. Thus, the specific structure of MAI is entirely ignored. MUD receivers are designed to take advantage of the knowledge of MAI signal parameters, albeit at the cost of increased complexity.

The following sections give a brief overview of such receivers, ranging from the original impractical optimal scheme to the more recent IC schemes that led to the proposed trickle model.

\subsubsection{Optimal Detection}

Based on the work of Van Etten [41] published in the mid-1970's, Verdu [2] first introduced the optimal multi-user receiver for CDMA in 1986. The receiver structure is based on maximum-likelihood (ML) detection, minimizing the squared Euclidean distance between the received signal and the sum of $K$ asynchronous user signals. Joint sequence decisions are made utilizing $K$ MF outputs as opposed to successive decisions stemming from each MF 


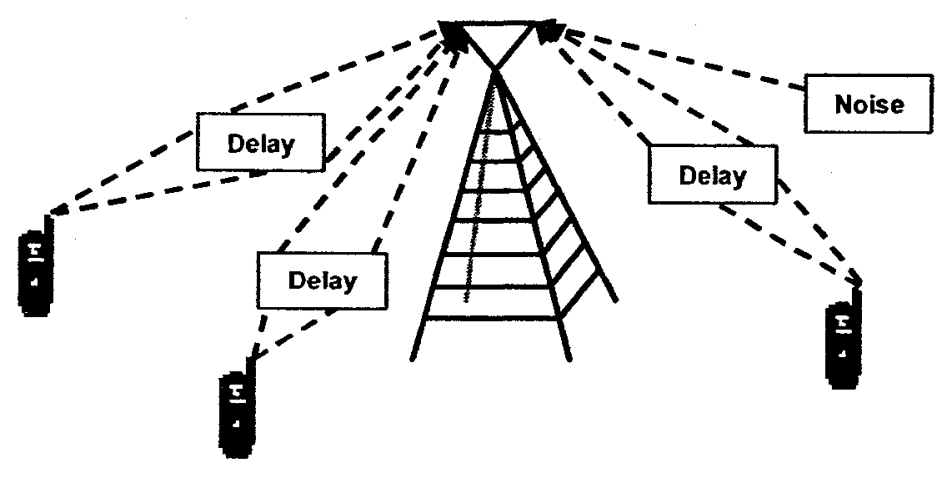

Figure 2.4: Uplink transmission at base station receiver

output alone. Although Verdu's optimal receiver achieved significant gains in performance, it unforunately suffered from severe complexity limitations, which increased exponentially with the number of users and the length of the sequence. What followed was a widerange of suboptimal designs geared towards reducing complexity while maintaing acceptable standards of performance. The role of the optimal receiver has been that of a benchmark against which to compare these sub-optimal CDMA receivers. The focus of this section is specifically on SIC, PIC, and hybrid-IC type receiver structures, where contrary to other types of MUD receivers, error correction coding is integrated into the MUD process.

\subsection{SIC}

\subsubsection{Conventional SIC}

One of the two main classes of multi-user interference cancellation receivers, SIC involves individual processing of $K$ users where interference is cancelled successively. The users are first ordered depending on their average signal strengths, the strongest user being the first to be detected. Each user's transmitted data is estimated and reconstructed in conjunction with 
the corresponding channel estimates. The reconstructed signal is then subtracted from the received signal, rendering the signal more reliable for subsequent users. All users experience improvement in performance; earlier users due to their high received power, and later users because a large fraction of the total interference has been removed prior to their detection. This process is illustrated below in Fig. 2.5

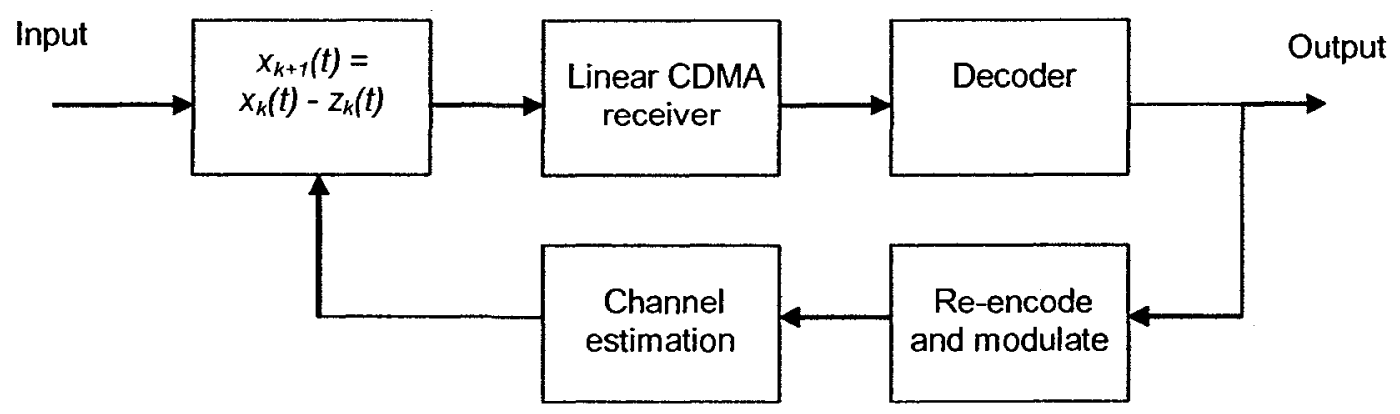

Figure 2.5: Successive Interference Cancellation

The complexity and latency of SIC receivers are proportional to $K$. Simulation plots for SIC for both $K=5$ users and $K=25$ users in comparison with the standard MF receiver are shown below in Fig. 2.6 and Fig. 2.7, respectively. It should be noted, that the results presented involve a large number of interferring users and the systems, each as a whole, involve more than the mere $K=5$ or $K=25$ users being processed.

SIC for $K=25$ users demonstrates superior BER performace as more accurate channel estimates are obtained for weaker users, albeit once again at the cost of increased latency. With fewer users, not only are fewer channel estimates utilized in the cancellation process, those very same estimates are relatively inaccurate to begin with [5].

\subsubsection{Soft-decision SIC}

Since low-power users' decisions are dependent on the accuracy of the interference cancellation and channel estimations obtained from the high-power users, a soft-decision approach to SIC was presented by [14] aimed at increasing the reliability of the decoding and estimation from te strongest users. Metrics for the stronger users are updated by subtracting the subsequent decisions on low-power users given that the high-power signal corresponding to each path in a trellis is reencoded and subtracted from the composite signal. In other words, to make a decision for a high-power user, decisions on a given number of subsequent low-power users are also considered through a series of hypothesis trellis paths. The process suffers from 
Figure 2.6: BER plot of SIC receiver for $K=5$.

increased overall latency and was presented merely as a conceptual scheme. Nonetheless, it provided a basis for the more practical and refined algorithms that followed [18],[20].

\subsubsection{Power Control for SIC}

For uplink transmission, the received powers from mobiles that are closer to the base station represent a larger portion of the total interference as compared to mobiles that are farther away. Conventional power control techniques to eliminate this problem by maintaining equal received powers for all users. Thus, users at the cell edges actually transmit at unecessarily higher powers, creating added interference for neighboring cells. The work of [17] introduced the concept of power control in accordance with successive interference cancellation schemes that rely on controlled disparities between the powers of users. The improvement in performance is achieved by appropriately enforcing disparate powers amongst users and allowing users at cell edges to be received at lower power levels. The CDMA capacity is thus affected by inter-cell interference only. 
Figure 2.7: BER plot of SIC receiver for $K=25$.

Further research [25]-[27] on the improvement of the power control algorithm demonstrated that higher capacity could be achieved by increasing the relative power of later users as the channel estimation error increases. This counters the residual interference originating from imperfect interference cancellation assumed otherwise by optimal received power distribution.

\subsection{PIC}

\subsubsection{Conventional PIC}

PIC is the second of the two main classes of multi-user interference cancellation receivers. Contrary to SIC, PIC detects all $K$ users simultaneously, providing a decrease in latency at the cost of less accurate channel estimation. A coarse initial estimate is obtained through joint estimation from a bank of $K$ matched filters and is used to cancel a portion of the interference. The parallel detection is then repeated for a given $S$ number of stages until 
sufficient interference cancellation is achieved. Thus, each user is processed $S$ times albeit in conjuction with all other users. The process is shown in Fig. 2.8

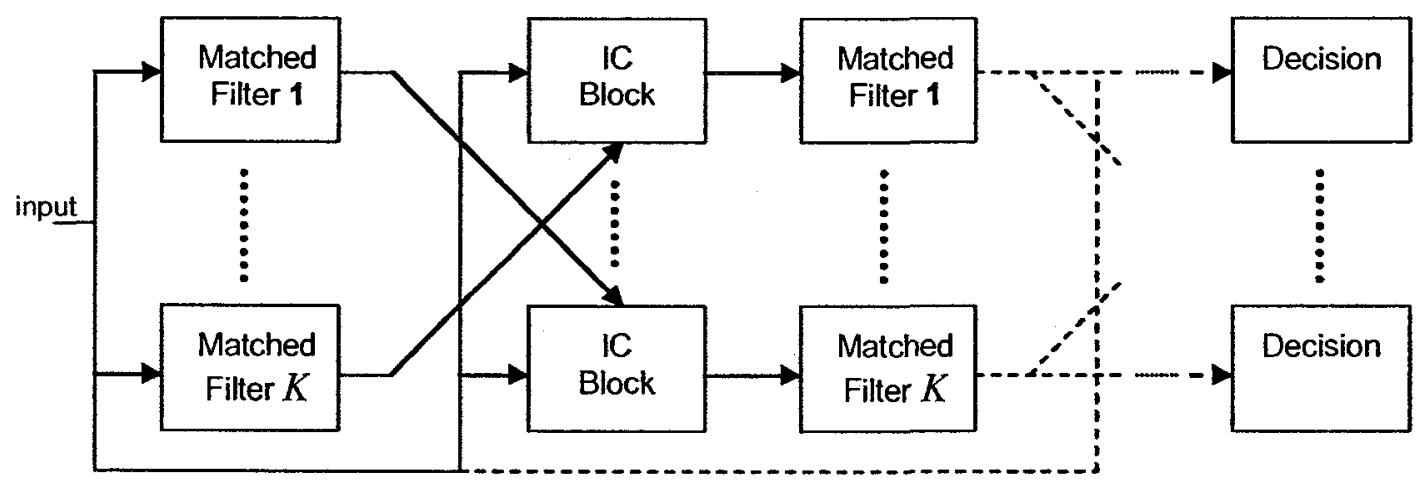

Figure 2.8: Parallel Interference Cancellation

The complexity of PIC receivers is proportional to $S \times K$ while the latency depends solely on $S$. Simulation plots for single and 3-stage PIC for $K=20$ users in comparison with the standard MF receiver are shown below in Fig. 2.9 and Fig. 2.10, respectively.

\subsubsection{Partial PIC}

The joint estimation obtained from the initial stages of the PIC process generally results in highly noisy data estimates. Thus, [15] presented a soft interference cancellation algorithm termed "partial parallel interference cancellation" (PPIC). PPIC attempts to counter system degradation by cancelling varying amounts of interference depending on reliability and number of iterations completed. The motive behind this approach lies in the low reliability of channel estimates performed in earlier stages. Naturally, the negative influence a weak estimate is accentuated when complete interference cancellation is involved. Partial removal of interference allows for a greater margin of error regarding inaccurate channel estimates when dealing with many users simultaneously; multiple iterations affect a weight factor that adjusts the amount of interference to be cancelled at each stage. The improvement lies in the process by which previous bit estimates are included in subsequent estimations, thus improving channel estimates for following iterations. The complexity of the system is linear to the number of users implicated. 
Figure 2.9: BER plot for single-stage PIC for $K=20$.

\subsubsection{Frequency Diversity Combining with PPIC}

The advent of PPIC created a new point of origin for much of the subsequent research regarding PIC receivers. One such example lies in the addition of frequency diversity combining (FDC) to PIC, and subsequently PPIC presented in [24], which offers better performance while maintaining similar complexity. The motivation for this approach is to merely achieve a higher diversity gain at minimal cost. FDC-PPIC is an extension of FDC-PIC and offers a more viable option for future IC applications.

\subsubsection{Iterative PIC}

The work of [28] investigates an iterative approach to joint estimation. This work was motivated by the promising results shown in previous work on iterative decoding strategies [10]-[29], most notably for CDMA systems. The receiver is designed to infer a probability distribution on the code symbols for each user as well as compute channel estimates in an 
Figure 2.10: BER plot for 3-stage PIC for $K=20$.

iterative fashion. Each of the two sets of information are updated for every stage of the detection process, leading to improved overall system performance due to the convergence of the estimates.

The iterative decoding receiver is, however, limited by a critical user load, under which near single user performance is obtained. If the critical load is exceeded, the receiver is rendered ineffective. Furthermore, the bank of detectors assigned to the users implicated can be processed in parallel or in successive fashion, thus extending the receiver's applications to various IC receivers [30].

\subsection{Hybrid IC}

More recently, hybrid-type MUD receivers have been proposed combining benefits inherent to both PIC and SIC schemes. While there are limitations inherent to both approaches, significant gains can be achieved when they are applied in conjunction with one another. 


\subsubsection{Concatenated IC}

A most primitive and obvious, yet effective hybrid design was first presented by [11]. The structure of the scheme consists of several individual receivers each incorporating both a serial and three parallel cancellation stages. The motivation for such a design lies in the potential improvement in overall system performance made possible by utilizating both SIC and PIC to obtain channel estimates. The first serial cancellation stage is used to obtain initial data estimates while the subsequent parallel stages are used to enhance the accuracy of the estimates and carry out the interference cancellation process. Groups are detected in parallel yet early estimates are obtained via SIC. A flowchart of the steps involved in the process of concatenated IC receivers is shown in Fig. 2.11.

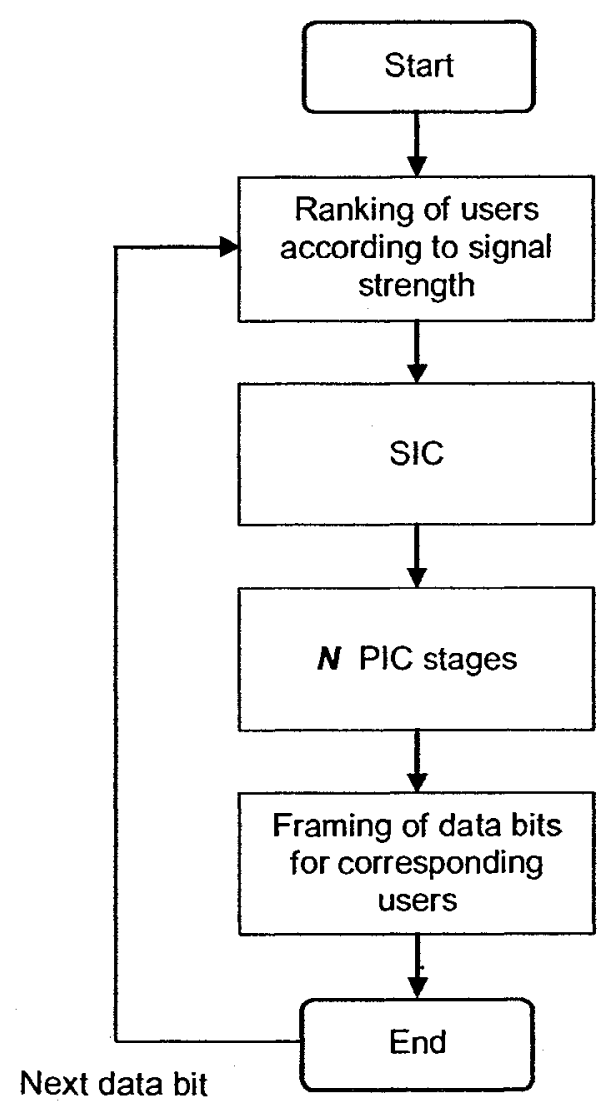

Figure 2.11: Flowchart of serial-parallel receiver model

The structure can accomodate a large number of users offerring more versatility than other hybrid designs described in the following section. 


\subsubsection{Groupwise IC}

Group-wise successive interference cancellation (GSIC), one of the early hybrid designs, utilizes channel estimation to cancel interference in groups [6], [17]. The system is setup much like standard SIC systems, save the group-wise configuration. The motive behind such an approach lies in the great gain in hardware complexity which is reduced by a factor equal to the group size. However, due to the propagation of the negative effects of inaccurate channel estimation, GSIC cannot achieve the performance of SIC. It therefore becomes a question of trade-off and specific design requirements rather than a brute performance comparison.

Improvement on such designs is achieved by means of the iterative receiver principle mentioned above. The iterative approach suggested by [33] yielded two novel groupwise structures. Iterative group-wise parallel interference cancellation (IGPIC) and iterative groupwise serial interference cancellation (IGSIC) demonstrate how various configurations of users can lead to gains in performance at the cost of latency. The performance of IGPIC is in fact considered superior to that of IGSIC. Users are organized into groups increasing in size by one user per stage culminating in $K$ total stages where the final group consists of $K$ users. Users are ranked according to their signal strength prior to being configured into groups, resulting in the strongest user being processed individually to begin, and and at every subsequent stage thereafter. A sample configuration of the scheme for $K=5$ users is shown below in Fig. 2.12

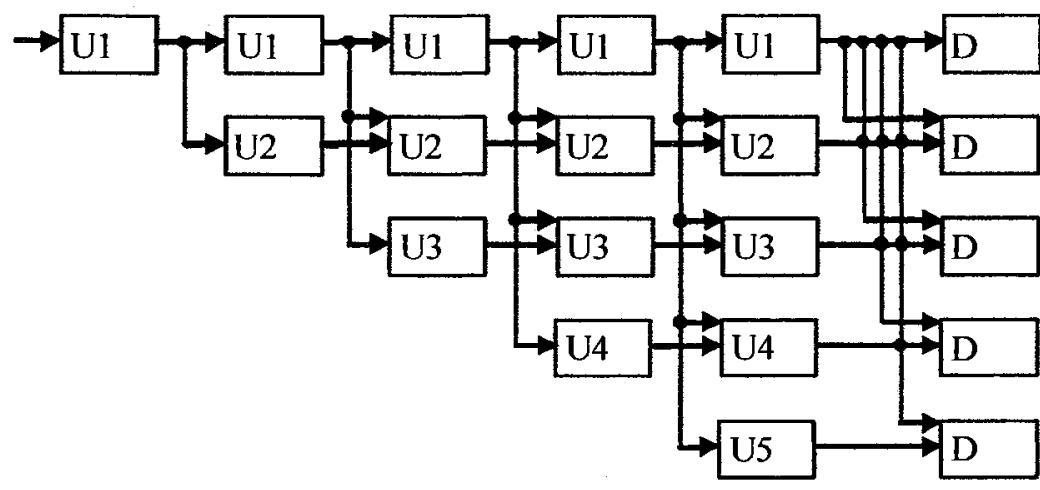

Figure 2.12: Block diagram of group-wise interference cancellation scheme for $K=5$.

Interference is cancelled out simultaneously for all users of a given stage, the resulting composite signal of which is passed on to the next stage as is the case with conventional PIC operation. Unlike conventional PIC, each stage carries a greater number of users as the IC 
process is carried out. This results in an improvement in performance for all users; those in earlier groupings due to high SINR, and those in later groupings where a large fraction of the total interference is removed prior to detection. Groupwise IC offered a new approach for MUD receivers by means of altered user configuration benefitting from both successive and parallel processing of users.

\subsection{Introduction to Pipelined Multiuser Detection}

As mentioned in the previous sections, the focus of much of the research concerning MUD is to combine different IC methods in order to take advantage of various benefits as well as overcome the limitations inherent to the schemes in question. The proposed scheme employs pipelined MUD, the detailed description of which is presented in the following sections. However, it should be noted that despite the advantages offered by this scheme, its inclusion is arbitrary and other schemes with similar benefits can be used in its stead. Its description is nonetheless significant in that its benefits are reflected in the results obtained.

\subsubsection{Background to the Partial Parallel Pipelined Multiuser De- tector}

The work of [21] focuses on the different system requirements for both the channel estimation and detection processes. The high computational complexity associated with asynchronous multiuser detection methods led to the proposed iterative based suboptimal scheme, designed to eliminate the need for matrix inversions and avoid multishot detection for schemes where initial estimates are obtained via a matched filter. By implementing a bit-streaming architecture, stages of the iteration are "pipelined", resulting in a reduction in latency. The following sections describe this structure in greater detail.

\subsubsection{Channel Estimation and Pipelined Multistage Detector}

The channel estimation process is similar to most multiuser detectors. A pilot sequence of bits known at the receiver is utilized in order to determine channel effects regarding delays and amplitudes. These channel parameters are extracted from the signal at the receiver by comparing the received bits with the known pilot sequence. Maximum likelihood (ML) estimation is employed, and the channel parameters are assumed to remain static until the end of the frame. These estimates are then passed on to the pipelined multistage detector where interference cancellation is performed stage by stage. 


\subsubsection{Matched Filter and Pipelined Multistage Detector Overview}

The detector utilizes both the MF outputs and the cross-correlation information of all users in the system. The first stage in the baseband signal detection consists of a bank of matched filters, each assigned to a single user in the system. Alternatively to (2.5), the matched filter output $y$ for a given user is represented by

$$
y=\mathbf{R A} d+\eta
$$

where $d$ is the transmitted data bits, $\mathbf{R}$ is the cross correlation matrix of the synchronized spreading codes, $\mathbf{A}$ is a diagonal matrix containing the amplitudes of the users, and $\eta$ is the AWGN. The structure of $\mathbf{R}$ is given by

$$
\mathbf{R}=\mathbf{I}+S+S^{T}
$$

where $\mathbf{I}$ is the identity matrix and $S$ is the lower triangular portion of the matrix $\mathbf{R}$.

The structure of $\mathbf{A}^{H} \mathbf{A}$, described in [21], is given by

$\left[\begin{array}{ccccc}\mathbf{A}_{0}^{H} \mathbf{A}_{0} & \mathbf{A}_{0}^{H} \mathbf{A}_{1} & 0 & 0 & 0 \\ \mathbf{A}_{1}^{H} \mathbf{A}_{\mathbf{0}} & \mathbf{A}_{0}^{H} \mathbf{A}_{0}+\mathbf{A}_{1}^{H} \mathbf{A}_{\mathbf{1}} & \mathbf{A}_{0}^{H} \mathbf{A}_{\mathbf{1}} & 0 & 0 \\ 0 & \mathbf{A}_{1}^{H} \mathbf{A}_{0} & \mathbf{A}_{0}^{H} \mathbf{A}_{0}+\mathbf{A}_{1}^{H} \mathbf{A}_{\mathbf{1}} & \mathbf{A}_{0}^{H} \mathbf{A}_{\mathbf{1}} & 0 \\ : & \ddots & \ddots & \ddots & : \\ 0 & 0 & 0 & \mathbf{A}_{1}^{H} \mathbf{A}_{0} & \mathbf{A}_{0}^{H} \mathbf{A}_{0}+\mathbf{A}_{1}^{H} \mathbf{A}_{\mathbf{1}}\end{array}\right]$,

where $D$ is the number of received bits, and $K$ is the number of users. As mentioned previously, it is assumed that the channel remains static during the detection window. This leads to the tri-diagonal block-Toeplitz nature [21] of the $\mathbf{A}^{H} \mathbf{A}$ matrix shown above, allowing for pipelined detection. A more in-depth analysis of this detector is given in [22].

\subsubsection{Detailed System Model Description}

In vector form, the received signal is given by 


$$
r_{i}=\left[\mathbf{A}_{0} \mathbf{A}_{1}\right]\left[\begin{array}{c}
d_{1, i-1} \\
: \\
d_{K, i-1} \\
d_{1, i} \\
: \\
d_{K, i}
\end{array}\right]+\eta_{i}
$$

where the data bits $d$ of the $K$ users to be detected lie between the received signal $r_{i}$ and $r_{i-1}$ boundaries. Lower index $i$ represents time. Thus, the matched filter detects each user individually, ignoring the effects of other users. The hard decision of the MF detector is given by

$$
\hat{d}_{i}=\operatorname{sgn}\left(\Re\left[\mathbf{A}_{1}^{H} r_{i-1}+\mathbf{A}_{0}^{H} r_{i}\right]\right) .
$$

The causal nature of the matched-filter detector means it does not require succeeding bits of other users to produce an output estimate [21]. The PIC component of the detector is performed in stages for the multistage detector [3], [31] and requires multishot detection. It should be noted that the proposed hybrid model incorporates PIC albeit for varying group sizes, and the pipelined multistage detector remains applicable. Multishot detection is the cancelling of the interference caused by the succeeding bits of users and is modeled as

$$
r_{i}=\left[\begin{array}{cccc}
\mathbf{A}_{0} & \mathbf{A}_{1} & 0 & 0 \\
0 & \mathbf{A}_{0} & \mathbf{A}_{1} & 0 \\
: & \ddots & \ddots & \mathbf{A}_{1} \\
0 & 0 & 0 & \mathbf{A}_{0}
\end{array}\right]\left[\begin{array}{c}
d_{1,1} \\
: \\
d_{K, 1} \\
d_{1, D} \\
: \\
d_{K, D}
\end{array}\right]+\eta_{i}
$$

where $r$ is the multishot received vector of dimension $N \times D, N$ being the spreading factor and $D$ being the number of received bits. Here, $A$ represents the new multishot channel matrix. The initial soft and hard decision outputs obtained from the matched filter are respectively given by

$$
y^{(0)}=\Re\left[\mathbf{A}^{H} r\right]
$$

and 


$$
d^{(0)}=\operatorname{sgn}\left(y^{(0)}\right)
$$

During multi-user detection, a given user's bits are affected by the interference of previous and subsequent overlapping symbols from other users as well as their current symbols due to the asynchronous nature of the signals. The pipelined effect is illustrated below in Fig. 2.13

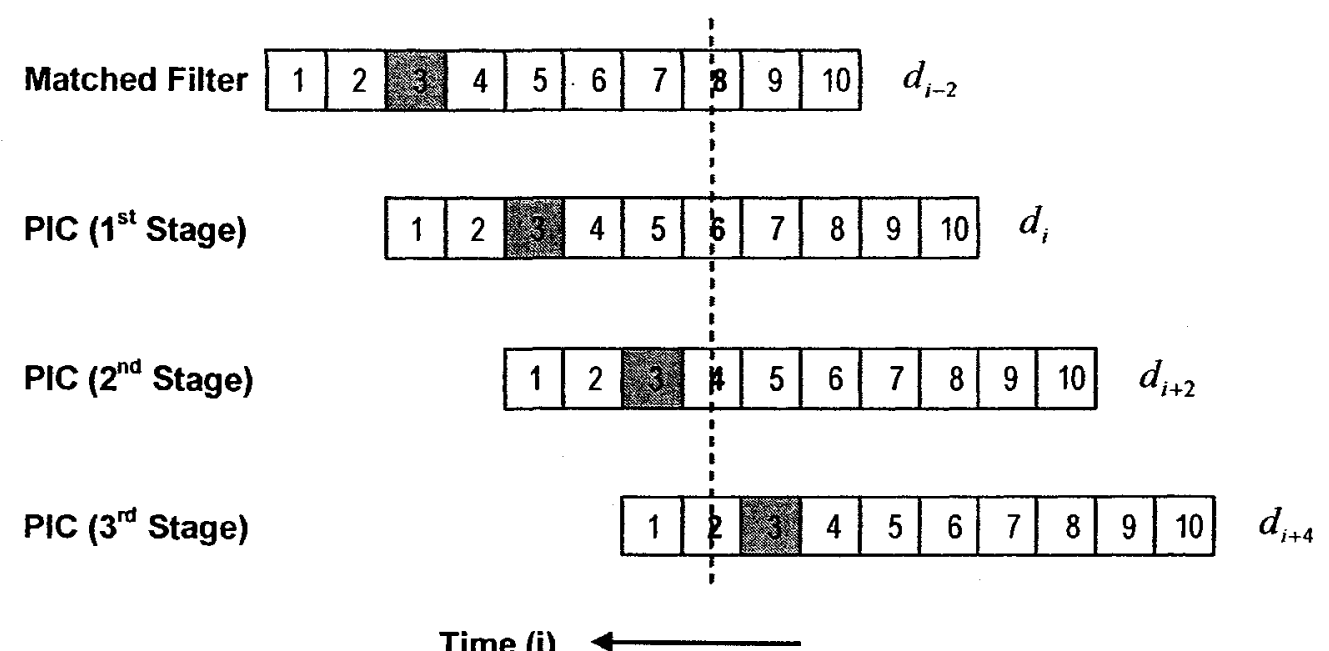

Figure 2.13: Pipelined detection process

The detection of bit 3 is featured as an arbitrary example. An initial estimate of the received signal is obtained via the MF detector, which depends solely on current and past received bits. As mentioned above, bits 2 and 4 of all users are needed in order to cancel the interference for bit 3 . Therefore, the first PIC stage can only begin processing bit 3 after the estimates of bits 2 and 4 are obtained. Similarly, the second PIC stage, in turn, can only begin after bit 4 is processed by the previous stage, and so on. This leads to a reduction in latency which, for the purposes of this study, are irrelevant and will not be looked at in detail. The motive of this scheme as well as a more in depth analysis of its benefits can be found in [22]

The computations performed on the intermediate bits reduce to

$$
y_{i}^{(l)}=y_{i}^{(0)}-\mathbf{L} \hat{d}_{i-1}^{(l-1)}-\mathbf{C} \hat{d}_{i}^{(l-1)}-\mathbf{L}^{T} \hat{d}_{i+1}^{(l-1)},
$$


and

$$
\hat{d}_{i}^{(l)}=\operatorname{sgn}\left(y_{i}^{(l)}\right)
$$

where $y_{i}^{(l)}$ and $\hat{d}_{i}^{(l)}$ are once again the respective soft and hard decisions obtained after each stage of the multistage detector, lower index $i$ once again represents time, and the upper index $l$ represents the number of IC iterations. More specifically, $y_{i}^{(l)}$ describes the interference being subtracted due to the past and future bits of interferering users, where $\mathbf{L}$ is the partial correlation matrix for the past and future bits of the interfering users given by

$$
\mathbf{L}=\Re\left[\hat{\mathbf{A}}_{1}^{H} \hat{\mathbf{A}}_{0}\right]
$$

and $\mathbf{C}$ is the center matrix representing the correlation of the current bits of interfering users given by

$$
\mathbf{C}=\Re\left[\hat{\mathbf{A}}_{0}^{H} \hat{\mathbf{A}}_{0}+\hat{\mathbf{A}}_{1}^{H} \hat{\mathbf{A}}_{1}-\operatorname{diag}\left(\hat{\mathbf{A}}_{0}^{H} \hat{\mathbf{A}}_{0}+\hat{\mathbf{A}}_{1}^{H} \hat{\mathbf{A}}_{1}\right)\right]
$$

where the diagonal elements are made zeros as only the interference from other users, represented by the non-diagonal elements, need to be canceled.

The entire multiuser detection process is shown below in Fig. 2.14.

All simulations of the proposed algorithm incorporate the abovementioned pipelined MUD architechture. Once again, it should be noted that this MUD model is chosen arbitrarily; similar results can be achieved utilizing other existing models for channel estimation. 


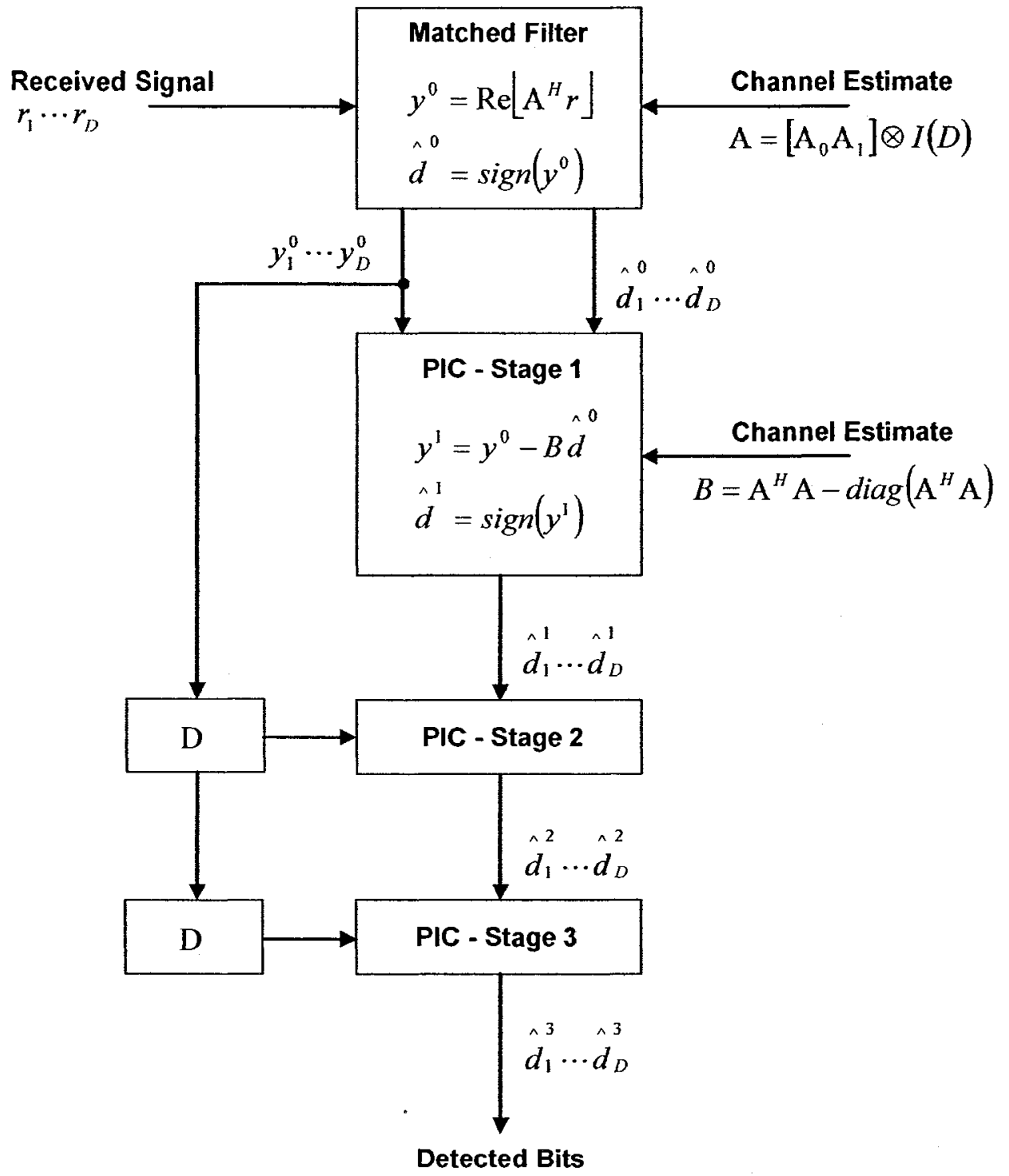

Figure 2.14: Multistage multiuser detection overview 


\section{Chapter 3}

\section{Proposed Scheme - The Trickle Algorithm}

\subsection{Derivation of the Trickle Algorithm}

The proposed trickle algorithm incorporates principles of both SIC and PIC schemes. As with conventional SIC, users are ordered depending on the average signal strength. Interference is cancelled in stages successively, each stage consisting of a certain number of users determined by the applied algorithm and the corresponding total number of users. PIC is performed at every stage regardless of the number of users.

Let $r_{p}(t)$ represent the composite input signal to the $p$ th stage. As with standard PIC, a given stage processes a signal resulting from the subtraction of the original received signal by the estimated MAI from all previous stages. For instance, when $p=2$, the signal is given by

$$
r_{2}(t)=r(t)-\hat{s}_{1}\left(t-\hat{\tau}_{1}\right)
$$

One of the major limiting factors of the joint estimation involved in PIC receivers is the detrimental contributions by users with weaker signal strength. SIC can overcome this by ordering users according to their signal power and processing users individually, obtaining reliable channel estimates from the strongest user first, the next strongest user second, and so forth. Thus, subsequent users benefit from the reliable early estimates despite their weaker signal. However, the advantages of joint estimation are lost as users contribute to channel estimation accuracy only once.

It is both advantageous to process certain users repeatedly and counter-productive to process other users more than once. The proposed algorithm employs PIC at every stage, 
but contrary to standard PIC operation, not all users are included in every stage of the IC process. Joint estimation is utilized, albeit for various numbers of users per group. The initial stages include the strongest users exclusively in order to achieve improved joint estimation. Two options for such a design are presented in the following section.

\subsection{Exponentially Varying Group Sizes}

Each interference cancellation stage produces an estimate of the signal that is carried over to the next group of users. In order to take advantage of the more reliable early estimates, initial stages can be made to include fewer users, more specifically those exhibiting stronger signal power. This results in varying group sizes amongst different interference cancellation stages. However, channel estimation does not improve linearly with every stage. Thus, it is counter-intuitive to arrange users in linearly varying group sizes. Therefore, we increase the number of users in a non-linear manner. Through analysis presented below, it is shown that schemes employing non-linearly varying group sizes produce better BER performance with comparable processing delay as compared to similar linear models as well as standard PIC structures.

In this section, we investigate two novel hybrid IC schemes. The resulting analysis provides the basis for the proposed trickle algorithm. Both schemes employ exponentially varying group sizes. The first involves a greater number of users in the early stages, attempting to take advantage of joint estimation to establish reliable early estimates. Latter stages increase in size, but in inverse exponential fashion. The second model begins with a single user found to be strongest and therefore most reliable, and increases the number of users exponentially for each stage until the $K$ users are processed. The number of additional users per stage for the inverse exponential and exponential models are given respectively by

$$
U=\text { floor }[K /(2 s)]
$$

and

$$
U=2^{s-2}
$$

where $U$ is the number of users to be added to a given stage and $s$ is the index of the given stage starting from the second stage, $s=2$. Equations (3.2) and (3.3) are set as guidlines for the two hybrid models. They are arbitrary and are used merely as a means to compare two different strategies for preliminary channel estimation. The first model is designed to benefit 
from the performance properties of the standard PIC scheme by first employing groupwise IC stages. The second model follows SIC principles during early IC stages, utilizing individual processing of users in order to obtain more accurate early estimates. Both models demonstrate identical system latency as the number of total stages is maintained. Sample configurations of both models for $K=8$ users are shown in Fig. 3.1 and Fig. 3.2.

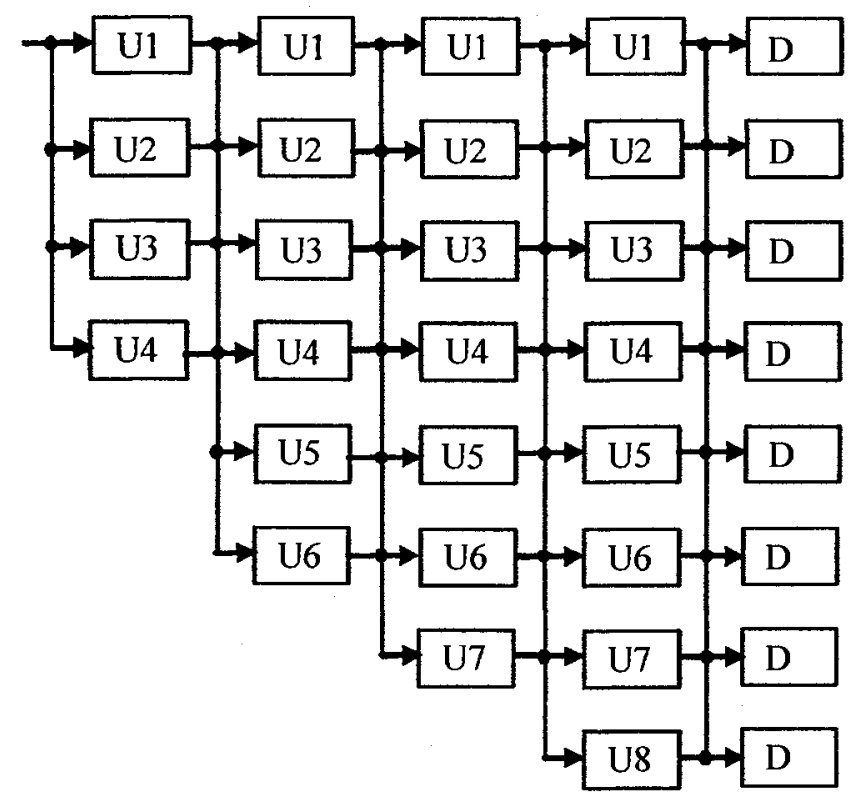

Figure 3.1: Block diagram of inverse exponential group-wise scheme for $K=8$.

The terms exponential and inverse exponential refer to the manner in which the number of users are increased as compared to the previous stage. For the configuration shown in Fig. 3.1, the number of users per stage increases in an inverse-exponential fashion, while the configuration in Fig 3.2 demonstrates an exponential increase.

Consider the initial stages of the inverse exponential scheme. Some of the users involved exhibit low signal power but are nonetheless implicated in the joint estimation process. The positive effect of the more diverse joint estimation is, to a certain extent, countered by a negative influence attributed to a relatively weak signal strength associated with these users. For higher values of SNR, there is a greater benefit in obtaining phase and amplitude estimates from a single reliable user rather than through joint estimation of many users. This correlates with the comparisons made between the performance of SIC and PIC when ignoring drawbacks regarding overall system latency. Comparatively, the exponential groupwise structure offers improved channel estimation for higher values of SNR and consequently 


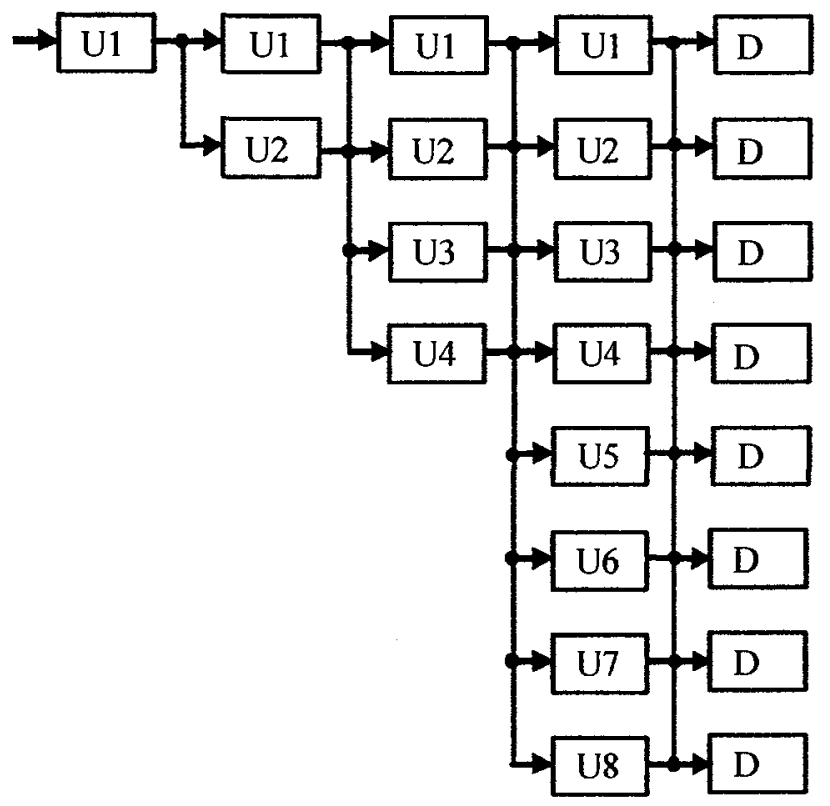

Figure 3.2: Block diagram of exponential group-wise scheme for $K=8$.

demonstrates better BER performance. Simulation results supporting the abovementioned benefits are presented in Section 4. However, estimation can be further improved through additional iterations for stronger users, giving rise to the proposed trickle scheme described in the following section.

\subsection{Description of the Trickle Algorithm}

Motivated by similar factors as the exponential and inverse exponential group-wise schemes, the trickle algorithm employs non-linearly varying group sizes determined by the total number of users. A count matrix $\mathbf{Q}$ of dimension $K \times 2 K$ is created in order to compute the number of IC iterations required for all users. Each row corresponds to one user and each column represents one IC stage. The users are ordered in a decreasing fashion according to their signal strength such that the top rows of the matrix represent the stronger users and the bottom rows the weaker ones. The system is first modeled as an upper-right triangular matrix thus replicating the appropriate processing pattern for $K$ number of users as in [33]. Secondly, the last iterations from each of the first $u$ rows are removed and redistributed amongst the first $v$ rows where 


$$
u=(K-1)-2 i, \quad i=0,1, \ldots, w,
$$

and

$$
v=\text { floor }(K / 2)
$$

where $w$ is the number of redistributions carried out by the trickle algorithm and is governed by

$$
w=\text { floor }(\operatorname{ceil}(K / 2) / 2)
$$

There are $u$ iterations to be redistributed amongst $v$ rows with the value of $u$ changing with every repetition of the process denoted by $i$. Equation (3.4) is created as such in order to ensure that the last column carries out detection for all users for any given value of $K$. It is also designed to limit the increase in the number of users to two for the latter columns of the matrix. Thus, joint estimation at a given stage involves no more than two additional users as compared to the previous stage, guarding against degredation in channel estimation which can arise when several users with weak signal strength are being processed simultaneously for the first time. For example, if a given stage carries five more users than the previous stage, the resulting channel estimation is affected by five potentially unreliable users and the negative contributions are propagated throughout the IC process. This limitation leads to better overall channel estimation and offers improved reliability over the exponential groupwise scheme shown in Fig. 3.2.

The value of $v$ described in (3.5) is set such that the users with the strongest signal power receive the greatest number of redistributed iterations. In other words, the rows located in the top half of the $\mathbf{Q}$ matrix receive all redistributions and the corresponding users are consequently processed more often than those situated in the bottom half.

The redistribution process begins at row $v$ and continues through to row 1 , each row successively acquiring $j$ supplementary iterations for $j=1,2, \ldots, v^{\prime}$ where

$$
v^{\prime}=u-\left((v-1)^{2}+v-1\right) / 2
$$

The reliability of early channel estimation is futher increased through this process. Equation (3.7) ensures that the upper rows within the top half of the $\mathbf{Q}$ matrix receive even more iterations than those closer to row $v$. More explicitly, row $v$ gains one iteration, row $v-1$ gains two, and so forth. For the final step, Equation (3.7) merely adds the remaining $v^{\prime}$ 

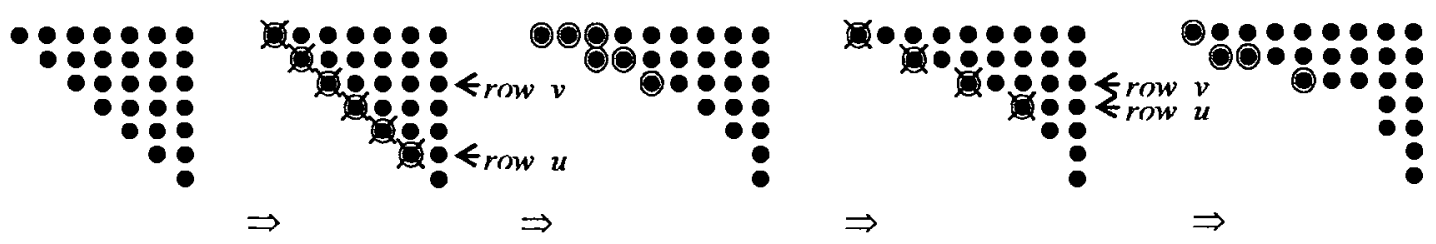

$\Rightarrow$

Figure 3.3: Sample Q matrix computation for 7 users.

iterations left over from the original total $u$ to row 1 . For certain values of $K$, however, this can lead to a row 1 having fewer iterations than row 2 . Once all redistributions are carried out, an inversion of the two first rows is added if necessary to ensure that the strongest user is in fact relied on more heavily than all other users.

The resulting matrix, denoted by $\tilde{\mathbf{Q}}$, represents the trickle pattern applicable to the desired MUD detection scheme. It is important to note that the expressions describing $u, v$, and $w$ are derived through experimentation and are optimized with regards to the proposed algorithm. They are computed as such in order to ensure a proper reconfiguration of users for any given value of $K$. Furthermore, for a given $K$, the $\tilde{\mathbf{Q}}$ matrix is unique and identical for every realization since its construction depends solely on $K$, not on the users' signal strength. Thus, the computation of $\tilde{\mathbf{Q}}$ does not add to the system's overall processing delay as it can be pre-configured for any value of $K$.

In this example, we consider the execution of the trickle algorithm for $K=7$ users. The corresponding computation of the $\tilde{Q}$ matrix is shown in detail in Fig. 3.3. The following pseudocode describes the process:

- Create a count matrix $\mathbf{Q}$ of dimension $K \times 2 K$ : A matrix containing 7 rows and 14 columns is created.

- Remove the first iterations from each of the first $u$ rows and redistribute amongst the first $v$ rows: From Equations (3.4) and (3.5), $i=0, u=(7-1)-2 \times 0=6$, and $v=$ floor $(7 / 2)=3$. In other words, the last iterations from rows 6 through 1 are eliminated and are inserted back into the matrix staring from row 3 through to the first row.

- The redistribution of iterations starts from row $v=3$. The algorithm adds $j$ supplementary iterations to each row, where $j=1,2, \ldots, v^{\prime}$ : From Equation (3.7), $v^{\prime}=6-\left((3-1)^{2}+3-1\right) / 2=3$. Thus, rows 3 through 1 receive $j=1,2$, and 3 iterations, respectively.

- The redistribution process is repeated $w$ times: From Equation $(3.6), w=$ floor $(\operatorname{ceil}(7 / 2) / 2)=$ 
2. For $K=7$, redistribution is therefore executed only twice. The second and final execution is carried out with $i=1, u=(7-1)-2 \times 1=4$, and $v^{\prime}=4-\left((3-1)^{2}+3-1\right) / 2=$ 1. The value of $v$ remains the same as it does not depend on $i$. In this final redistribution, $u=4$ iterations are removed and rows $v=3$ through 1 receive $j=1,2$, and 1 iterations, respectively.

- If the number of total iterations is greater for user 2 than user 1 , invert rows 1 and 2 : This step is not carried out for $K=7$ as row 1 does not contain fewer iterations than row 2.

\subsection{Improved Trickle Algorithm}

As mentioned above, each row of the $\tilde{\mathbf{Q}}$ matrix represents a single user while each column represents an individual PIC stage along with the number of users invovled. Certain columns are repeated many times, resulting in greater latency while failing to improve the BER performance. This is due to the negligible gains achieved by executing PIC for more than three iterations. In other words, typical convergence of standard PIC operation occurs after three successive stages and, as shown in [16], improvement in the BER performance is greatly reduced following a third PIC run. By limiting each distinct grouping to a maximum of three repetitions in the $\tilde{\mathbf{Q}}$ matrix, the BER performance is maintained and the overall processing delay is reduced.

The configuration of the reduced matrix remains dependent on $K$ and is therefore once again consistent for any given value of $K$. The improved algorithm does not increase the complexity as, similar to the original trickle scheme, matrix configurations can be constructed and stored prior to the detection process.

Also, the larger the number of users, the greater the number of reduced stages. Repeated columns occur more often when the redistribution process is carried out more frequently. Thus, the gains in performance for the improved trickle algorithm become especially significant for systems with larger values of $K$.

For this example, let us consider the original $\tilde{\mathbf{Q}}$ matrix for $K=21$ users shown in Fig. 3.4 .

As can be seen, there are five distinct groupings that repeat for more than three stages. More specifically, columns containing one, three, four, five, and six users appear more than three times. The improved trickle algorithm reduces these groupings to a maximum of three stages by removing the excess columns from the original $\tilde{\mathbf{Q}}$ matrix. The columns to be 


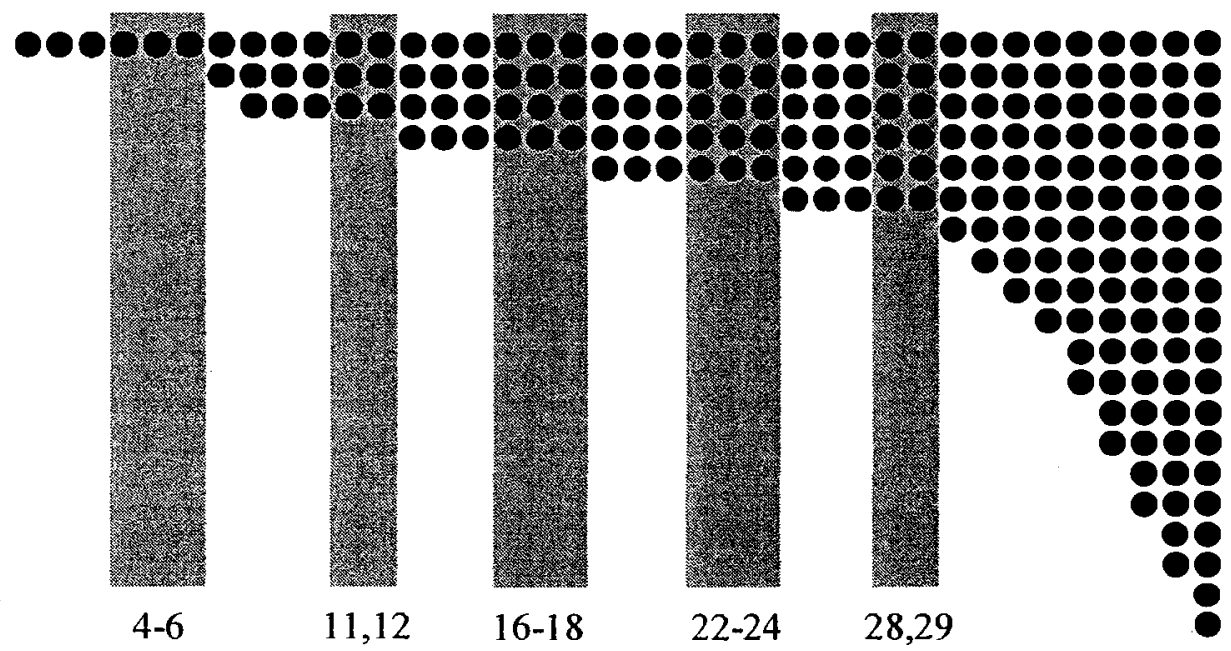

Figure 3.4: Sample Q matrix for $K=21$.

removed are highlighted and numbered in Fig. 3.4. The resulting reduced $\widetilde{\mathbf{Q}}$ matrix is shown in Fig. 3.5.

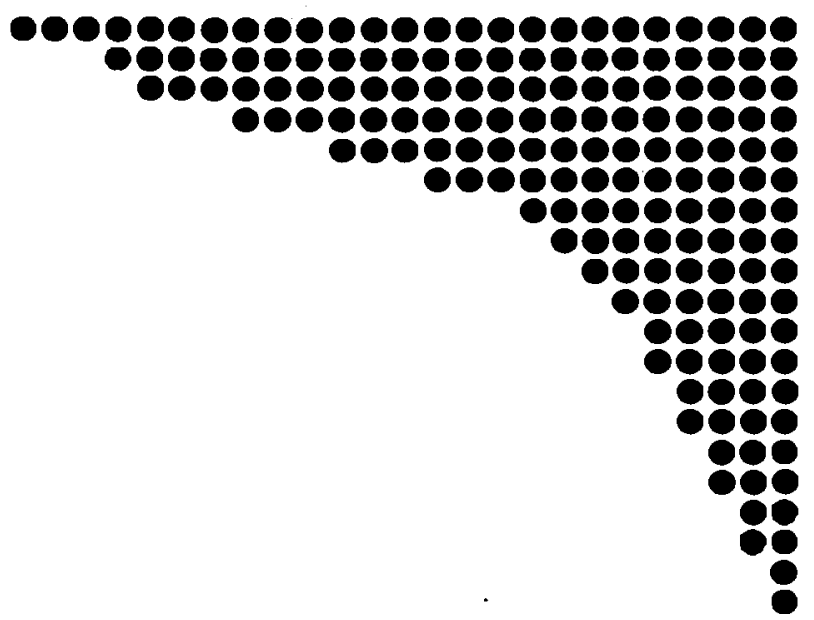

Figure 3.5: Reduced Q matrix for $K=21$.

When comparing Figs. 3.4 and 3.5, there occurs a reduction in the number of columns from 38 to 25 . Once again, each column represents an IC stage and a reduction in stages correlates directly with a reduction in system latency. For $K=21$, the improved trickle algorithm achieves a $(38-25) \div 38=34.2 \%$ reduction in total IC stages. A more detailed analysis of the gains in processing delay is provided in the next chapter. 


\section{Chapter 4}

\section{Results}

We consider the uplink channel of a CDMA system. The standard number of users is set to $K=20, K=60$, and $K=120$ for various simulation runs, each user being assigned a spreading code of length $C=32$. BPSK modulation is employed and the spreading factor is set to $N=32$. The system incorporates $L_{P}=3$ multipaths with uniform power delay profile. Pilot data generated from a predetermined sequence known at the receiver, sent with the same SNR as the data generated subsequently, is used to construct the estimation matrices described in Section 2.7. Short channel delay spread is assumed such that the $k$ th user bit interferes only with its adjacent neighboring bits. Furthermore, the channel parameters are assumed to remain static during the detection window. This prevents the necessity for the re-ordering of users and ensures the validity of the pre-configured $\tilde{\mathbf{Q}}$ matrix. For comparison purposes, indentical channel conditions are applied to all simulation runs.

\subsection{Exponential Versus Inverse Exponential}

Fig. 4.1 compares the performance of the two schemes involving exponentially varying group sizes discussed in Section 3. Simulation results validate theoretical expectations as the exponential scheme achieves superior BER performance for higher values of SNR. For SNR values greater than 12 , the exponential scheme experiences an improvement of approximately $1 \mathrm{~dB}$ over the inverse exponential scheme. Simulation results for $\mathrm{SNR}=18 \mathrm{~dB}$ and $K=$ 20 users further illustrates this effect. A greater improvement is demonstrated in channel estimates obtained from single users and small groups of users characterizing the initial stages of the exponential scheme. 
Figure 4.1: BER comparison between exponential and inverse exponential group-wise schemes for $K=20$.

\subsection{Performance Comparison between Trickle, Linear, Exponential, MF, PIC, and SIC}

Fig. 4.2 compares the performance of the proposed trickle algorithm to that of the standard MF receiver, both 3-stage PIC and SIC schemes, the hybrid receiver based on [11] consisting of linearly varying group sizes, as well as the superior of the two exponential models discussed above. Despite the increase in processing delay, the trickle algorithm greatly outperforms both SIC and PIC schemes. The trickle model also shows improvement over previous hybrid models, albeit to a much lesser degree when compared to the exponential scheme presented in Section 3. Despite similar structures, however, the proposed scheme offers better BER performance at the cost of increased latency due to the greater number of total IC stages. As can be seen, greater improvement is achieved at higher values of SNR. The results presented offer a means of comparison for uncoded systems and nonetheless demonstrate the potential 
gains with regards to coded systems. Furthermore, in order to provide a more accurate comparison to previously designed schemes, the system parameters are generated such that the near-far effect is accounted for. The advantage of the trickle algorithm lies in further establishing dependable early estimates such that the latter stages experience less corruption due to weaker users. At BER $10^{-3}$, the trickle MUD receiver exhibits an improvement of more than $1.5 \mathrm{~dB}$ over the linear model, and an improvement of $0.7 \mathrm{~dB}$ over the exponential scheme presented in section 3.2 .

Figure 4.2: BER comparison between linear, exponential, MF, SIC, 3-stage PIC, and trickle algorithm for $K=20$.

Simulation results for $K=60$ and $K=120$ are presented Fig. 4.3 and Fig. 4.4, respectively. Both figures demonstrate relatively consistent performance benefits over conventional MUD schemes, the linear hybrid model, as well as the experimental exponential scheme. Naturally, as the number of users is increased, BER performance slightly degrades due to the increase in MAI power [34]. 
Figure 4.3: BER comparison between linear, exponential, MF, SIC, 3-stage PIC, and trickle algorithm for $K=60$.

\subsection{Processing Delay Reduction Analysis}

Fig. 4.5 compares the BER performance of the improved trickle algorithm with that of the original model prior to the reduction process. As can be seen, the difference in performance is negligible and is far less significant relative to the gains in overall system latency. This is consistent with theoretical expectations concerning PIC receivers where exceeding three iterations does not generally produce significantly superior results.

Fig. 4.6 is a representation of the potential reduction in processing delay. Analysis of both the total number of IC stages as well as the total number of processing events is presented. By processing events we mean the total number of times all users are detected throughout the entire IC run. For example, for $K=7$ shown in Fig. 3.3, the number of processing events is 28 . The number of stages is reduced only when the trickle configuration produces a repitition of more than three IC stages for a given group size. This happens for the first time at $K=11$. The percent reduction in total number of IC stages increases with 
Figure 4.4: BER comparison between linear, exponential, MF, SIC, 3-stage PIC, and trickle algorithm for $K=120$.

the number of users, reaching over $40 \%$ for the case where $K=30$. It is once again important to note that the reduction achieved by the algorithm is identical for any realization of the algorithm as it depends on the number of users $K$. More specifically, the plot shown in Fig. 4.6 is universally true for all instances where the improved trickle algorithm is applied. The reduction in processing events illustrates the lack of degradation in the BER performance. By maintaining a sufficiently high number of processing events, the performance is also maintained. 
Figure 4.5: BER comparison between improved and standard trickle algorithmfor $K=20$. 
Figure 4.6: Percent reduction in number of IC stages and processing events for the improved trickle algorithm. 


\section{Chapter 5}

\section{Conclusion and Future Work}

\subsection{Conclusion}

In this thesis, we proposed a novel interference cancellation receiver structure for a CDMA uplink system. The model incorporates the proposed "trickle" algorithm, in order to reduce the effects of MAI during the detection process and achieve an improvement in BER performance. Two novel hybrid schemes employing exponentially varying group sizes were presented from which the trickle scheme was derived. Through analysis, it was shown that the exponential scheme achieved greater performance over the inverse exponential scheme, leading to the development of various parameters involved in the proper construction of an adaptive user configuration matrix utilized by the trickle algorithm.

We also demonstrated that the performance of the proposed scheme surpassed that of the conventional MUD schemes, the linear hybrid model, as well as the experimental exponential schemes. This was accomplished by relying more heavily on users with stronger signal strength in order to obtain early channel estimates.

Finally, a reduced matrix was subsequently developed and an improved trickle algorithm was presented. Through simulation and timing analysis, it was shown that the improved trickle algorithm was capable of providing similar BER performance while significantly reducing overall system processing delay as compared to the original trickle algorithm.

\subsection{Future work}

In the following, we point out several related directions of research.

- The next and most logical step of our work is to apply the proposed trickle algorithm to WCDMA uplink transmission. Incorporating appropriate scrambling codes into 
the pipelined MUD receiver structure would render the algorithm more practical and relevant with regards to more up-to-date wireless standards.

- Throughout this study, we have assumed proper channel estimation prior to the interference cancellation process. The channel estimation of this scheme is obtained through the pipelined MUD via a pilot sequence of bits known at the receiver. However, we have not investigated the effects of improper channel estimation. Stronger estimation techniques, such as [22], [46] could be implemented and analyzed in order to achieve superior performance to that of the current scheme. In addition, improved power control algorithms can be applied [25], further enhancing the advantages of emploing the trickle matrix.

- More complex adaptations of the trickle algorithm could also be investigated. A groupwise trickle configuration could improve BER performance by dividing the $\tilde{\mathbf{Q}}$ matrix into subgroups, each containing a condensed version of the $\tilde{\mathbf{Q}}$ matrix, maintaining the basic rules of construction. As such, the users with weaker signal strength are generally more dependant on those with stronger signal strength since each subgroup contains fewer IC stages.

- The principle of the $\tilde{\mathbf{Q}}$ matrix could also be applied to groups of users, each element of the $\tilde{\mathbf{Q}}$ matrix consisting of multiple users. This configuration would be advantageous for systems with a large number of users where a significant reduction in processing delay is achieved due to the fewer number of IC stages involved. However, this configuration suffers in BER performance as a less accurate portion of the interference is cancelled prior to given individual stages. The study would investigate the trade-off between decreased latency versus decreased performance.

- The application of multiple antenna schemes could provide additional benefits in terms of array gain, diversity gain, and interference reduction [47]. The next step would involve analysis of the effects of an antenna array at the base station, and subsequent implementation of several models in order to determine the optimal configuration with regards to the proposed trickle scheme. Furthermore, a multiple antenna system can potentially achieve drastic improvement in performance and system latency in combination with the abovementioned group-wise trickle scheme. The IC process could be divided amongst different antennas provided that a proper recombining algorithm is designed. 


\section{Bibliography}

[1] Mats Nilsson, "Third generation radio access standards," Ericsson Review, pp. 110-121, Nov. 1999.

[2] S. Verdu, "Minimum probability of error for asynchronous Gaussian multiple-access channels," IEEE Trans. Inform. Theory, vol. IT-32, pp.85-96, Jan. 1986.

[3] M. K. Varanasi and B. Aazhang, "Multistage detection in asynchronous code-division multiple-access communications," IEEE Trans. Commun., vol. 38, pp. 509-519, Apr. 1990.

[4] S. Moshavi, "Multi-user detection for DS-CDMA communications," IEEE Commun. Mag., vol. 34, pp.124-137, Oct. 1996.

[5] P. Patel and J. Holtzman, "Analysis of a simple successive interference cancellation scheme in a DS/CDMA," IEEE J. Select. Areas Commun., vol. 12, pp.769-807, May 1994.

[6] F. Wijk, G. M. J. Janssen, and R. Prasad, "Groupwise successive interference cancellation in a DS/CDMA system," Proc. IEEE PIMRC, pp. 742-746, 1995.

[7] T. R. Giallorenzi and S. G. Wilson, "Suboptimum multiuser receivers for convolutionally coded asynchronous DS-CDMA systems," IEEE Trans. Commun., vol. 44, no. 9, pp. 1183-1196, Sept. 1996.

[8] R. Muller and J. Huber, "Capacity of cellular CDMA systems applying interference cancellation and channel coding," in Proc. IEEE GLOBECOM, pp. 179-184, Nov. 1997.

[9] P. G. Renucci, "Optimization of soft interference cancellation in DS-CDMA receivers," Masters thesis, Virginia Polytechnic Institute \& State University, Blackburg, Virginia, 1998. 
[10] J. Hagenauer, "Forward error correcting for CDMA systems," IEEE Int. Symp. on Spread Spectrum Techniques and Applications, pp. 566-569, Sept. 1996.

[11] T-B. Oon, R. Steele, and Y. Li, "Performance of an adaptive successive serial-parallel interference cancellation scheme in flat Rayleigh fading channels," Proc. IEEE VTC, pp. 193-197, 1997.

[12] P. Alexander and A. Grant, "Iterative sequence estimation using unconstrained statespace," in Proc. IEEE Int. Symp. on Information Theory, p. 242, Aug. 1998.

[13] P. Alexander, A. J. Grant, and M. C. Reed, "Iterative detection on code-division multiple-access with error control coding," European Transactions on Telecommunications, vol. 9, no. 5, pp. 419-426, Sept.-Oct. 1998.

[14] M. Brandt-Pearce, M.-H. Yang, "Soft-decision multiuser detector for coded CDMA systems," IEEE Trans. Commun., vol. 1, pp. 365-369, June 1998.

[15] D. Divsalar, M. Simon, and D. Raphaeli, "Improved parallel interference cancellation for CDMA," IEEE Trans. Commun., vol. 46, no. 2, pp. 258-68, Feb. 1998.

[16] D. Guo, L. K. Rasmussen, and T. J. Lim, "Linear parallel interference cancellation in long-code CDMA multiuser detection," IEEE J. Select. Areas Commun., vol. 17, pp. 2074-2081, Dec. 1999.

[17] D. Warrier and U. Madhow, "On the capacity of cellular CDMA with successive decoding and controlled power disparities," Proc. IEEE VTC, pp. 1873-1877. May 1998.

[18] L. K. Rasmussen, T. J. Lim, A. Johansson, "A matrix-algebraic approach to successive interference cancellation in CDMA," IEEE Trans. Commun., vol. 48, no. 1, pp. 145-151, Jan. 2000.

[19] H.-M. Kang and S. H. Cho, "Multiuser detection algorithms for CDMA," Tech Report, Communications and Signal Processing Laboratory, Hanyang University, Seoul, South Korea, 2001

[20] W. Zha, S.D. Blostein, "Soft-decision successive interference cancellation CDMA receiver with amplitude averaging and robust to timing errors," IEEE Trans. Commun., vol. 2, pp. 743-747, Nov. 2001. 
[21] S. Rajagopal and J.R. Cavallaro, "A bit-streaming, pipelined multiuser detector for wireless communication receivers," Proc. IEEE ISCAS, Sydney, Australia, vol. 4, pp. 128-131, May 2001.

[22] S. Rajagopal et al., "Real-time algorithms and architectures for multiuser channel estimation and detection in wireless base-station receivers," IEEE Trans. Wireless Commun., vol. 1, no. 3, pp. 468-79, July 2002.

[23] H. Schoeneich and P. A. Hoeher, "Single antenna interference cancellation: iterative semi-blind algorithm and performance bound for joint maximum-likelihood interference cancellation," Proc. IEEE GLOBECOM, San Francisco, CA, pp. 1716-1720, Dec. 2003.

[24] G. Xu and L. Gan, "Partial parallel interference cancellation for multi-carrier DSCDMA," IEEE Trans. Commun., vol. 1, pp. 623-26, Sept. 2005.

[25] J. G. Andrews and T. Meng, "Optimum power control for successive interference cancellation with imperfect channel estimation," IEEE Trans. Wireless Commun., vol. 2, no. 2, pp. 375-383, Mar. 2003.

[26] J. G. Andrews and T. Meng, "Performance of MC-CDMA with successive interference cancellation in a multipath fading channel," IEEE Trans. Commun., vol. 52, no. 5, pp. 811-822, May 2004.

[27] R. M. Buehrer, "Equal BER performance in linear successive interference cancellation for CDMA systems," IEEE Trans. Commun., vol. 49, no. 7, pp. 1250-1258, July 2001.

[28] P. Alexander and A. J. Grant, "Iterative channel and information sequence estimation in CDMA," Proc. IEEE Int. Symp. on Spread Spectrum Techniques and Applications, pp. 593-597, Sept. 2000.

[29] X. Wang and H. V. Poor, "Iterative (turbo) soft nterference cancellation and decoding for coded CDMA," IEEE Trans. Commun., vol. 47, no. 7, pp. 1046-1061, July 1999.

[30] M. Kobayashi, J. Boutros, and G. Caire, "Successive interference cancellation with SISO decoding and EM channel estimation," IEEE JSAC, vol. 19, no. 8, pp. 1450-1460, Aug. 2001.

[31] G. Xu and J. R. Cavallaro, "Real-time implementation of multistage algorithm for next generation wideband CDMA systems," Advanced Signal Processing Algorithms, Architectures.and Implementations IX, SPIE, Denver, Colorado, vol. 3807, pp. 62-73, July 1999. 
[32] R. Malik, V.K. Dubey, and B. McGuffin, "A hybrid interference canceller for CDMA systems in Rayleigh fading channels," IEEE Vehicular Technology Conference, VTC 2001 Spring. IEEE VTS 53rd, vol. 2, pp. 1523-1527, 2001.

[33] J. Koo, "Performance analysis of an iterative group-wise parallel interference cancellation for multiuser detection of coherent W-CDMA system," Young-In SongDam College, 2002.

[34] B. Manatsavee, K Ahmed, and A. Fernando, "Performance of PIC, SIC, and decorrelating detectors for MUD technique in WCDMA," Proc. IEEE ICICS, Singapore, pp. 892-896, December 2003.

[35] A. Agrawal, J. Andrews, J. Cioff, and T. Meng, "Iterative power control for imperfect successive interference cancellation," IEEE Trans. Wireless Commun., vol. 4, no. 3, pp. 878-884, May 2005.

[36] P. Tan and L. K. Rasmussen, "Belief propagation for coded multiuser detection," IEEE Int. Symp. Inf. Theory, Seattle, USA, pp. 1919-1923, July 2006.

[37] Y. Hara, L. Brunel, and K. Oshima, "Downlink spatial scheduling with mutual interference cancellation in multiuser MIMO systems," Proc. IEEE PIMRC, Sep. 2006.

[38] V. Tikiya, S. Manohar, and A. Chockalingam, "SIR-optimized weighted linear parallel interference canceller on fading channels," IEEE Trans. Wireless Commun., vol. 5, no. 8, pp. 1998-2003, Aug. 2006.

[39] Y.-T. Hsieh and W.-R. Wu, "Optimal two-stage decoupled partial PIC receivers for multiuser detection," IEEE Trans. Wireless Commun., vol. 4, no. 1, pp. 112-127, Jan. 2005.

[40] R.A. Scholtz, "The origins of spread spectrum communications," IEEE Commun. Magazine, pp. 82 - 85, May 1982.

[41] W. Van Etten, "Maximum likelihood receiver for multiple channel transmission systems," IEEE Trans. Commun., vol. 24, no. 2, pp. 276-283, Feb. 1976.

[42] A. J. Viterbi, "Very low rate convolutional codes for maximum theoretical performance of spread spectrum multiple-access channels," IEEE JSAC, vol. 8, pp. 641-649, May 1990. 
[43] T. S. Rappaport, Wireless Communications Principles and Practice, Prentice Hall PTR, 1996.

[44] M. Moher and T. A. Gulliver, "Crossentropy and iterative decoding," IEEE Trans. Inform. Theory, vol. 44, no. 7, pp. 3097-3104, Nov. 1998.

[45] H. Holma and A. Toskala, CDMA for UMTS, 3rd ed., Wiley, 2002.

[46] R.A. Iltis and S. Kim, "Geometric derivation of expectation-maximization and generalized successive interference cancellation algorithms with applications to CDMA channel estimation," IEEE Trans. Signal Processing, vol. 51, no. 5, pp. 1367 - 1377, May 2003.

[47] C. Papadias, "On the spectral efficiency of space-time spreading schemes for multiple antenna CDMA systems," Conference Record of the Thirty-Third Asilomar Conference on Signals, Systems, and Computers, vol. 1, pp. 639 - 643, Oct. 1999. 